INTER NATIONAL MONETARY FUND
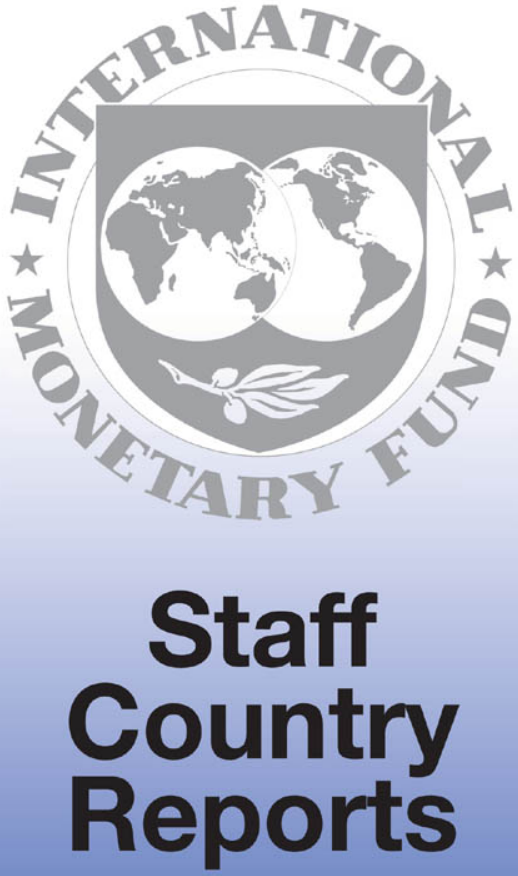
August 1999

IMF Staff Country Report No. 99/93

\section{Philippines: Statistical Appendix}

This Statistical Appendix report on Philippines was prepared by a staff team of the International Monetary Fund as background documentation for the periodic consultation with this member country. As such, the views expressed in this document are those of the staff team and do not necessarily reflect the views of the Government of Philippines or the Executive Board of the IMF.

Copies of this report are available to the public from

International Monetary Fund • Publication Services 70019 th Street, N.W. • Washington, D.C. 20431

Telephone: (202) 623-7430 • Telefax: (202) 623-7201

Telex (RCA): 248331 IMF UR

E-mail: publications@imf.org

Internet: http://www.imf.org

Price: $\$ 15.00$ a copy

International Monetary Fund

Washington, D.C. 


\section{INTERNATIONAL MONETARY FUND}

\section{PHILIPPINES}

\section{Statistical Appendix}

Prepared by Vivek Arora, Piyabha Kongsamut, Athanasios Vamvakidis (all APD), Enrique de la Piedra (MAE), Charalambos Christofides (PDR), and Kristina Kostial (FAD)

Approved by Asia and Pacific Department

June 28, 1999

Contents

Page

Selected Economic Indicators, 1993-98

Social and Demographic Indicators

$\underline{\text { Tables }}$

1. Gross National Product by Expenditure and Industrial Origin at Constant 1985 Prices, 1993-98

2. Gross National Product by Expenditure and Industrial Origin at Current Market Prices, 1993-98 .......................................................6

3. Gross Value Added in Manufacturing by Industry Group, 1993-98 _..............7

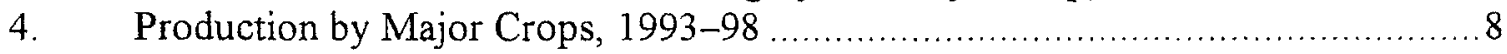

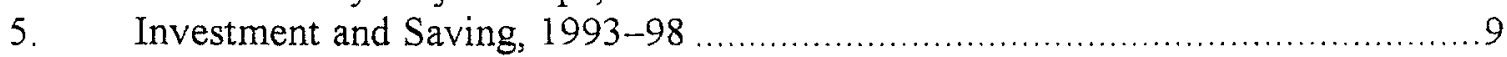

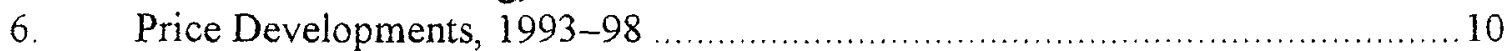

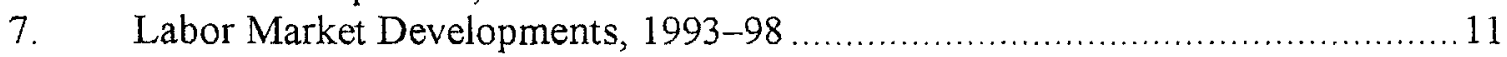

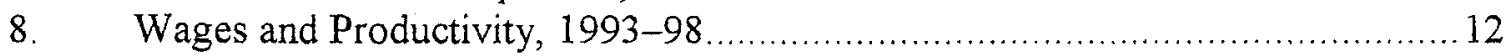

9. Domestic Retail Prices of Petroleum Products, 1993-98 ..............................13

10. Consolidated Public Sector Balance, 1993-98 .........................................14

11. Operations of the National Government, 1993-98 ..................................15

12. National Government Revenues and Grants, 1993-98 .............................16

13. National Government Expenditures and Net Lending, 1993-98 _..................17

14. Operations of Social Security Institutions, 1993-98 ................................18

15 Local Governments' Budgetary Operations, 1993-98 ................................19

16. Public Sector Infrastructure Program, 1993-98 .......................................20

17. Statement of Financial Operations of 14 Major Monitored Public Corporations, 1993-98.

18. Cash Flows of 14 Major Public Corporations, 1993-98 ..............................22

19. Public Sector Operating and Maintenance Expenditures by

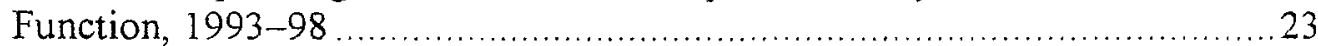

20. Functional Classification of National Government Expenditures, 1993-98 _.....24

21. Military Expenditure of the National Government, 1993-98 …....................25 
Philippines: Selected Economic Indicators, 1993-1998

Nominal GNP (1998): $\$ 71$ billion

Population (1998): 73.5 million

GNP per capita (1998): \$966

\begin{tabular}{|c|c|c|c|c|c|c|}
\hline & 1993 & 1994 & 1995 & 1996 & 1997 & 1998 \\
\hline \multicolumn{7}{|l|}{ GNP and prices (percentage change) } \\
\hline Real GNP & 2.1 & 5.2 & 4.9 & 7.2 & 5.2 & 0.1 \\
\hline Real GDP & 2.1 & 4.4 & 4.7 & 5.8 & 5.2 & -0.5 \\
\hline CPI (annual average) $1 /$ & 7.6 & 9.1 & 8.1 & 8.4 & 5.9 & 9.7 \\
\hline $\mathrm{CPI}$ (end year) $1 /$ & 8.4 & 7.2 & 11.0 & 5.2 & 7.3 & 10.3 \\
\hline \multicolumn{7}{|l|}{ Investment and saving (percent of GNP) } \\
\hline Gross investment & 23.6 & 23.5 & 21.8 & 23.1 & 23.8 & 19.3 \\
\hline National saving & 18.1 & 19.0 & 17.5 & 18.6 & 18.7 & 21.1 \\
\hline \multicolumn{7}{|l|}{ Public finances (percent of GNP) } \\
\hline National Government balance 2/ & -1.6 & -1.7 & -1.3 & -0.6 & -0.7 & -2.6 \\
\hline Total revenue & 17.2 & 18.4 & 17.9 & 18.1 & 19.2 & 16.9 \\
\hline Total expenditure and net lending & 18.8 & 20.1 & 19.2 & 18.6 & 19.9 & 19.5 \\
\hline Underlying consolidated public sector balance & -2.2 & -2.3 & -1.4 & -0.1 & -1.5 & -3.1 \\
\hline \multicolumn{7}{|l|}{ Monetary sector (percentage change, end of period) } \\
\hline Broad money (M3) & 24.6 & 26.5 & 25.3 & 15.8 & 20.9 & 7.4 \\
\hline Base money & 14.5 & 10.9 & 23.6 & 14.2 & 16.1 & 3.9 \\
\hline Interest rate ( $9 \mathrm{l}$-day Treasury bill, in percent) & 12.5 & 12.7 & 11.7 & 12.4 & 18.0 & 13.4 \\
\hline Bank credit to private sector & 37.6 & 28.2 & 43.5 & 51.0 & 28.7 & -3.1 \\
\hline \multicolumn{7}{|l|}{ External Sector (percentage change) } \\
\hline Export value & 15.8 & 18.5 & 29.4 & 17.8 & 22.8 & 16.9 \\
\hline Import value & 21.2 & 21.2 & 23.7 & 20.8 & 14.0 & -18.8 \\
\hline Current account (percent of GNP) & -5.5 & -4.5 & -4.3 & -4.6 & -5.1 & 1.9 \\
\hline Financial account (US\$ billions) & 2.0 & 4.1 & 4.1 & 8.0 & 0.9 & 0.1 \\
\hline Foreign direct investment (net) & 0.9 & 1.3 & 1.4 & 1.3 & 1.1 & 1.6 \\
\hline Other & 1.2 & 2.8 & 2.7 & 6.7 & -0.2 & -1.5 \\
\hline Overall balance (US\$ billions) & -1.0 & 1.1 & 0.8 & 4.1 & -3.4 & 1.4 \\
\hline Monitored external liabilities (percent of GNP) 3/ & 62.0 & 56.4 & 49.6 & 48.1 & 59.3 & 75.5 \\
\hline Debt-service ratio (percent) $4 /$ & 22.9 & 20.4 & 16.2 & 15.5 & 11.9 & 12.8 \\
\hline Reserves, adjusted (months of imports) $5 /$ & 2.5 & 2.7 & 2.2 & 2.8 & 1.7 & 2.7 \\
\hline \multicolumn{7}{|l|}{ Exchange rate } \\
\hline Nominal effective exchange rate $(1990=100) 6 /$ & 101.3 & 116.3 & 105.8 & 109.2 & 86.9 & 79.3 \\
\hline Real effective exchange rate $(1990=100) 6 /$ & 111.3 & 127.0 & 125.0 & 132.3 & 109.8 & 109.3 \\
\hline
\end{tabular}

Sources: Philippine authorities; Fund staff estimates; and IMF Information Notice System.

1/ CPI is 1994-based from 1997, while 1988-based for previous years.

2/ Fund definition. Excludes privatization receipts of the National Government, and includes net deficit from restructuring the central bank.

3/ For the years prior to 1997, the data refer only to external debt. For 1997 and later, they refer to a broader measure, which includes additional banking sector foreign liabilities.

4/ As a percentage of goods and services.

5/ Gross reserves less gold and securities pledged as collateral against short-term liabilities.

6/ End year. 
Philippines: Social and Demographic Indicators

\begin{tabular}{|c|c|c|}
\hline & $\begin{array}{c}15-20 \\
\text { years ago }\end{array}$ & $\begin{array}{l}\text { Most recent } \\
\text { estimate }\end{array}$ \\
\hline \multicolumn{3}{|l|}{ General } \\
\hline Land area (sq. km.) & 300,000 & 300,000 \\
\hline GNP per capita (U.S. dollars) & 340 & 966 \\
\hline Poverty incidence (percent) & 52 & 32.1 \\
\hline \multicolumn{3}{|l|}{ Income distribution (percent) } \\
\hline Lowest 20 percent & 5 & 6.5 \\
\hline Highest 20 percent & 56 & 47.8 \\
\hline \multicolumn{3}{|l|}{ Demographic } \\
\hline Population (million) & 42 & 71.9 \\
\hline Growth rate (percent) & 2.6 & 2.3 \\
\hline Population density (per sq. km.) & 144 & 241.1 \\
\hline Urban population (percent of total) & 35.6 & 54.9 \\
\hline Life expectancy (years) & 59.1 & 66.0 \\
\hline Crude birth rate (per '000 population) & 36.6 & 28.5 \\
\hline Crude death rate (per '000 population) & 9.7 & 6.6 \\
\hline \multicolumn{3}{|l|}{ Labor force } \\
\hline Total labor force (million) & 16 & 29.6 \\
\hline Agriculture (percent of total) & 53.3 & 39.0 \\
\hline Industry (percent of total) & 16.1 & 15.0 \\
\hline \multicolumn{3}{|l|}{ Education } \\
\hline Adult illiteracy (percent) & 16.7 & 5.4 \\
\hline Pupil/teacher ratio: primary school & 29 & 33.6 \\
\hline \multicolumn{3}{|l|}{ Enrollment (percent of age group) } \\
\hline Primary & 108 & 116 \\
\hline Secondary & 46 & 79 \\
\hline Tertiary & 24.4 & 27.4 \\
\hline \multicolumn{3}{|l|}{ Health } \\
\hline Infant mortality (per '000 live births) & 54.0 & 37.4 \\
\hline Population per physician & 9,100 & 8,424 \\
\hline Population per nurse & 2,691 & 2,684 \\
\hline \multicolumn{3}{|l|}{ Access to safe water } \\
\hline (percent of population) & 50 & 85 \\
\hline
\end{tabular}

Sources: World Bank; and data provided by the Philippine authorities. 
Table 1. Philippines: Gross National Product by Expenditure and Industrial Origin at Constant 1985 Prices, 1993-98

(Annual percentage changes)

\begin{tabular}{|c|c|c|c|c|c|c|}
\hline & 1993 & 1994 & 1995 & 1996 & 1997 & 1998 \\
\hline \multicolumn{7}{|l|}{ GNP by expenditure } \\
\hline Consumption & 3.3 & 3.9 & 4.0 & 4.6 & 4.7 & 3.3 \\
\hline Private & 3.0 & 3.7 & 3.8 & 4.6 & 5.0 & 3.5 \\
\hline Government & 6.2 & 6.1 & 5.6 & 4.1 & 1.6 & 0.8 \\
\hline Gross domestic investment & 7.9 & 8.7 & 3.5 & 12.5 & 11.7 & -17.1 \\
\hline Fixed investment & 8.7 & 7.5 & 4.7 & 12.0 & 11.5 & -10.7 \\
\hline Construction & 10.6 & 3.7 & 7.9 & 15.9 & 14.6 & -3.8 \\
\hline Durable equipment & 8.1 & 11.4 & 2.2 & 9.6 & 9.2 & -18.4 \\
\hline Breeding stock and orchard development & 1.9 & 2.6 & 3.9 & 6.0 & 8.5 & 0.2 \\
\hline Change in stocks & -30.7 & 94.1 & -44.1 & 45.5 & 24.5 & -351.6 \\
\hline Domestic demand & 4.2 & 4.9 & 3.7 & 6.4 & 6.3 & -1.6 \\
\hline Net exports $1 /$ & -2.5 & 0.5 & -2.8 & -2.3 & -0.2 & 1.7 \\
\hline Exports of goods and nonfactor services & 6.2 & 19.8 & 12.0 & 15.4 & 17.5 & -10.4 \\
\hline Imports of goods and nonfactor services & 11.5 & 14.5 & 16.0 & 16.7 & 14.4 & -11.4 \\
\hline \multicolumn{7}{|l|}{ GNP by industrial origin } \\
\hline Agriculture, fishery, and forestry & 2.1 & 2.6 & 0.9 & 3.8 & 2.9 & -6.6 \\
\hline Industry & 1.6 & 5.8 & 6.7 & 6.4 & 6.1 & -1.7 \\
\hline Mining and quarrying & 0.7 & -7.0 & -6.8 & 1.3 & 1.7 & 1.8 \\
\hline Manufacturing & 0.7 & 5.0 & 6.8 & 5.6 & 4.2 & -1.1 \\
\hline Construction & 5.7 & 8.9 & 6.5 & 10.9 & 16.2 & -8.1 \\
\hline Electricity, gas, and water & 2.9 & 13.9 & 13.0 & 7.5 & 4.8 & 4.4 \\
\hline Services & 2.5 & 4.2 & 5.0 & 6.4 & 5.5 & 3.5 \\
\hline Transportation, communication, and storage & 2.6 & 4.2 & 5.8 & 7.4 & 8.2 & 6.4 \\
\hline Trade & 2.5 & 4.0 & 5.6 & 5.5 & 3.9 & 2.4 \\
\hline Finance and housing & 2.0 & 4.0 & 4.9 & 8.3 & 8.0 & 3.0 \\
\hline Other private and government services & 2.8 & 4.8 & 4.1 & 5.4 & 4.0 & 3.7 \\
\hline Gross domestic product & 2.1 & 4.4 & 4.7 & 5.8 & 5.2 & -0.5 \\
\hline Net factor income from abroad $1 /$ & 0.04 & 1.38 & 0.35 & 2.28 & 0.35 & 0.6 \\
\hline Gross national product & 2.1 & 5.3 & 4.9 & 7.2 & 5.3 & 0.1 \\
\hline
\end{tabular}

Source: Data provided by the Philippine authorities.

1/ Contribution to real GNP growth. 
Table 2. Philippines: Gross National Product by Expenditure and Industrial Origin at Current Market Prices, 1993-98

(In billions of pesos)

\begin{tabular}{|c|c|c|c|c|c|c|}
\hline & 1993 & 1994 & 1995 & 1996 & 1997 & 1998 \\
\hline \multicolumn{7}{|l|}{ GNP by expenditure } \\
\hline $\begin{array}{l}\text { Consumption } \\
\text { Private } \\
\text { Government }\end{array}$ & $\begin{array}{r}1,272 \\
1,123 \\
149\end{array}$ & $\begin{array}{r}1,442 \\
1,259 \\
183\end{array}$ & $\begin{array}{r}1,629 \\
1,412 \\
217\end{array}$ & $\begin{array}{r}1,855 \\
1,595 \\
260\end{array}$ & $\begin{array}{r}2,082 \\
1,762 \\
320\end{array}$ & $\begin{array}{r}2,335 \\
1,980 \\
355\end{array}$ \\
\hline $\begin{array}{l}\text { Gross domestic investment } \\
\text { Fixed investment } \\
\text { Construction } \\
\text { Durable equipment } \\
\text { Breeding stock and orchard development } \\
\text { Change in stocks }\end{array}$ & $\begin{array}{r}354 \\
351 \\
149 \\
177 \\
25 \\
3\end{array}$ & $\begin{array}{r}407 \\
400 \\
165 \\
208 \\
27 \\
7\end{array}$ & $\begin{array}{r}428 \\
423 \\
184 \\
210 \\
30 \\
5\end{array}$ & $\begin{array}{r}522 \\
509 \\
231 \\
245 \\
33 \\
13\end{array}$ & $\begin{array}{r}602 \\
593 \\
270 \\
285 \\
38 \\
9\end{array}$ & $\begin{array}{r}541 \\
562 \\
270 \\
253 \\
39 \\
-20\end{array}$ \\
\hline Domestic demand & 1,625 & 1,849 & 2,057 & 2,376 & 2,684 & 2,876 \\
\hline $\begin{array}{l}\text { Net exports } \\
\text { Export of goods and nonfactor services } \\
\text { Imports of goods and nonfactor services }\end{array}$ & $\begin{array}{r}-125 \\
462 \\
587\end{array}$ & $\begin{array}{r}-107 \\
573 \\
679\end{array}$ & $\begin{array}{r}-149 \\
693 \\
842\end{array}$ & $\begin{array}{r}-191 \\
880 \\
1,071\end{array}$ & $\begin{array}{r}-251 \\
1,188 \\
1,439\end{array}$ & $\begin{array}{l}-100 \\
1,478 \\
1,578\end{array}$ \\
\hline Statistical discrepancy $1 /$ & -26 & -49 & -2 & -14 & -12 & -109 \\
\hline Gross domestic product & 1,474 & 1,693 & 1,906 & 2,172 & 2,421 & 2,667 \\
\hline Net factor income from abroad & 26 & 43 & 53 & 89 & 102 & 127 \\
\hline Gross national product & 1,500 & 1,736 & 1,959 & 2,261 & 2,523 & 2,794 \\
\hline \multicolumn{7}{|l|}{ GNP by industrial origin } \\
\hline Agriculture, fishery, and forestry & 319 & 373 & 412 & 448 & 453 & 450 \\
\hline $\begin{array}{l}\text { Industry } \\
\text { Mining and quarrying } \\
\text { Manufacturing } \\
\text { Construction } \\
\text { Electricity, gas, and water }\end{array}$ & $\begin{array}{r}482 \\
17 \\
350 \\
79 \\
36\end{array}$ & $\begin{array}{r}551 \\
17 \\
394 \\
95 \\
45\end{array}$ & $\begin{array}{r}611 \\
17 \\
438 \\
107 \\
49\end{array}$ & $\begin{array}{r}697 \\
17 \\
495 \\
128 \\
57\end{array}$ & $\begin{array}{r}780 \\
17 \\
540 \\
156 \\
66\end{array}$ & $\begin{array}{r}841 \\
20 \\
583 \\
160 \\
78\end{array}$ \\
\hline $\begin{array}{l}\text { Services } \\
\text { Transportation, communication, and storage } \\
\text { Trade } \\
\text { Financing and housing } \\
\text { Finance } \\
\text { Dwellings and real estate } \\
\text { Other } \\
\text { Private services } \\
\text { Government services }\end{array}$ & $\begin{array}{r}674 \\
78 \\
208 \\
157 \\
59 \\
99 \\
231 \\
127 \\
104\end{array}$ & $\begin{array}{r}770 \\
83 \\
231 \\
182 \\
67 \\
114 \\
274 \\
147 \\
127\end{array}$ & $\begin{array}{r}883 \\
89 \\
262 \\
209 \\
78 \\
130 \\
323 \\
169 \\
154\end{array}$ & $\begin{array}{r}1,027 \\
101 \\
295 \\
245 \\
96 \\
148 \\
386 \\
199 \\
187\end{array}$ & $\begin{array}{r}1,189 \\
119 \\
317 \\
282 \\
114 \\
168 \\
470 \\
234 \\
237\end{array}$ & $\begin{array}{r}1,376 \\
140 \\
361 \\
320 \\
130 \\
189 \\
556 \\
281 \\
275\end{array}$ \\
\hline Gross domestic product & 1,474 & 1,693 & 1,906 & 2,172 & 2,421 & 2,667 \\
\hline Net factor income from abroad & 26 & 43 & 53 & 89 & 102 & 127 \\
\hline Gross national product & 1,500 & 1,736 & 1,959 & 2,261 & 2,523 & 2,794 \\
\hline
\end{tabular}

Source: Data provided by the Philippine authorities.

1/ GDP by industrial origin minus domestic demand and net exports. 
Table 3. Philippines: Gross Value Added in Manufacturing by Industry Group, 1993-98

\begin{tabular}{|c|c|c|c|c|c|c|c|c|c|c|c|c|}
\hline Industry Group & 1993 & 1994 & 1995 & 1996 & 1997 & 1998 & 1993 & 1994 & 1995 & 1996 & 1997 & 1998 \\
\hline Food & 140.7 & 164.2 & 176.2 & 209.6 & 221.7 & 246.3 & -1.6 & 5.5 & 2.8 & 6.6 & 0.8 & 3.1 \\
\hline Beverage & 15.7 & 17.9 & 20.7 & 23.3 & 26.2 & 28.4 & -6.7 & 6.0 & 6.1 & 7.8 & 8.5 & -0.3 \\
\hline Tobacco & 9.1 & 10.1 & 10.6 & 11.6 & 12.8 & 13.5 & -3.6 & 3.8 & -0.7 & 8.0 & 5.9 & -4.2 \\
\hline Textiles & 10.5 & 10.0 & 11.2 & 11.2 & 11.1 & 11.9 & -0.7 & -7.4 & 6.3 & -2.0 & -3.2 & -3.8 \\
\hline Footwear and clothing & 25.2 & 27.1 & 30.4 & 29.6 & 31.5 & 36.5 & 7.1 & 5.5 & 8.1 & -8.8 & 2.7 & 3.7 \\
\hline Wood and cork products & 6.8 & 5.6 & 5.8 & 5.8 & 6.4 & 6.5 & 6.7 & -19.4 & 2.5 & -3.7 & 6.7 & -6.3 \\
\hline Furniture and fixtures & 5.2 & 5.9 & 6.9 & 7.1 & 8.2 & 8.9 & -3.8 & 6.9 & 11.3 & 2.0 & 11.8 & -0.1 \\
\hline Paper and paper products & 2.9 & 3.2 & 3.9 & 3.9 & 3.8 & 4.4 & -8.4 & 4.3 & 19.4 & -2.5 & -5.5 & 6.6 \\
\hline Publishing and printing & 4.5 & 5.0 & 5.5 & 6.0 & 6.6 & 6.7 & -6.2 & 4.6 & 6.3 & 1.3 & 3.8 & -4.3 \\
\hline Leather and leather products & 0.2 & 0.2 & 0.3 & 0.3 & 0.4 & 0.4 & -2.7 & 1.4 & 15.0 & 13.0 & 12.6 & 4.7 \\
\hline Rubber products & 3.8 & 3.8 & 4.4 & 4.3 & 4.0 & 3.8 & -13.7 & -4.2 & 11.8 & -8.2 & -8.2 & -11.4 \\
\hline Chemicals and chemical products & 28.9 & 31.2 & 35.7 & 39.2 & 44.8 & 49.1 & 3.3 & 1.3 & 8.1 & 5.8 & 7.3 & -0.4 \\
\hline Petroleum and coal products & 33.9 & 34.5 & 36.9 & 40.8 & 42.8 & 41.9 & -0.7 & 4.7 & 7.8 & 8.8 & 1.2 & -5.7 \\
\hline Nonmetallic mineral products & 11.7 & 14.1 & 17.1 & 19.6 & 22.8 & 20.6 & 9.8 & 10.7 & 14.1 & 7.5 & 12.6 & -16.5 \\
\hline Basic metals & 9.2 & 10.0 & 13.4 & 13.6 & 13.8 & 13.3 & 7.2 & 3.9 & 26.8 & -3.9 & -1.5 & -10.3 \\
\hline Metal products & 8.7 & 9.0 & 9.8 & 11.3 & 11.0 & 10.2 & 1.6 & -1.1 & 5.0 & 10.8 & -4.3 & -12.6 \\
\hline Machinery (except electrical) & 3.9 & 4.3 & 5.1 & 6.0 & 6.9 & 7.1 & 4.0 & 7.0 & 17.3 & 15.3 & 13.5 & -5.8 \\
\hline Electrical machinery & 16.0 & 22.7 & 28.1 & 33.7 & 45.6 & 53.0 & 14.9 & 22.7 & 13.5 & 14.7 & 31.1 & 6.5 \\
\hline Transportation equipment & 5.1 & 6.1 & 7.2 & 8.0 & 7.4 & 5.4 & 21.8 & 7.8 & 16.6 & 1.4 & -11.1 & -34 \\
\hline Miscellaneous manufactures & 7.3 & 8.9 & 9.3 & 10.5 & 12.7 & 15.2 & 8.9 & 13.3 & 2.2 & 6.4 & 13.2 & 9.6 \\
\hline Gross value added in manufacturing & 349.6 & 393.8 & 438.2 & 495.4 & 540.3 & 583.1 & 0.7 & 5.0 & 6.8 & 5.6 & 4.2 & -1.1 \\
\hline
\end{tabular}

Source: Data provided by the Philippine authorities. 
Table 4. Philippines: Production by Major Crops, 1993-98

(Area in thousands of hectares; yield in metric tons per hectare; and production in thousands of metric tons)

\begin{tabular}{|c|c|c|c|c|c|c|}
\hline & 1993 & 1994 & 1995 & 1996 & 1997 & 1998 \\
\hline \multicolumn{7}{|l|}{ Palay rice } \\
\hline Area under cultivation & 3,282 & 3,651 & 3,759 & 3,951 & 3,842 & 3,170 \\
\hline Yield & 2.9 & 2.9 & 2.8 & 2.9 & 2.9 & 2.7 \\
\hline Production & 9,434 & 10,538 & 10,541 & 11,284 & 11,269 & 8,555 \\
\hline Wholesale price (peso per kg.) & 10.78 & 12.11 & 15.07 & 17.38 & 16.89 & 17.39 \\
\hline \multicolumn{7}{|l|}{ Corn } \\
\hline Area under cultivation & 3,149 & 3,006 & 2,692 & 2,736 & 2,726 & 2,354 \\
\hline Yield & 1.5 & 1.5 & 1.5 & 1.5 & 1.6 & 1.7 \\
\hline Production & 4,798 & 4,519 & 4,128 & 4,151 & 4,332 & 3,823 \\
\hline \multicolumn{7}{|l|}{ Wholesale price (peso per $\mathrm{kg}$.) } \\
\hline Yellow & 5.60 & 6.20 & 7.40 & 7.68 & 7.64 & 8.32 \\
\hline White & 5.03 & 5.94 & 7.12 & 7.93 & 7.06 & 7.13 \\
\hline \multicolumn{7}{|l|}{ Fruit and nuts } \\
\hline Production & 8,695 & 8,892 & 8,981 & 9,287 & $\ldots$ & \\
\hline \multicolumn{7}{|l|}{ Coconut products } \\
\hline Area under cultivation & 3,075 & 3,083 & 3,064 & 3,149 & 3,314 & $\ldots$ \\
\hline Yield & 3.7 & 3.6 & 4.0 & 3.6 & 3.6 & $\ldots$ \\
\hline Copra price (peso per kg.) & 5.0 & 6.8 & 7.3 & 9.2 & 8.1 & 11.3 \\
\hline Production (copra equivalent) & 2,097 & 2,074 & 2,255 & 2,104 & 2,231 & 1,942 \\
\hline Domestic use & 2,516 & 2,490 & 2,706 & 2,651 & $\ldots$ & $\cdots$ \\
\hline \multicolumn{7}{|l|}{ Export (quantity in '000 MT) } \\
\hline Copra & 27 & 24 & 31 & 3.0 & 7.0 & 3.6 \\
\hline Coconut oil & 859 & 848 & 1,340 & 793 & 1,080 & 1,177 \\
\hline Desiccated coconut & 93 & 75 & 73 & 70 & 77 & 72 \\
\hline \multicolumn{7}{|l|}{ Centrifugal sugar } \\
\hline Area under cultivation & 384 & 402 & 302 & 396 & 352 & \\
\hline Farmgate price (peso per kg.) & 7.9 & 9.5 & 14.0 & 13.6 & 12.0 & 14.9 \\
\hline
\end{tabular}

Source: Data provided by the Philippine authorities 
Table 5. Philippines: Investment and Saving, 1993-98

(In percent of GNP)

\begin{tabular}{|c|c|c|c|c|c|c|}
\hline & 1993 & 1994 & 1995 & 1996 & 1997 & 1998 \\
\hline Gross domestic investment & 23.6 & 23.5 & 21.8 & 23.1 & 23.8 & 19.3 \\
\hline Public & 5.7 & 4.8 & 4.5 & 4.4 & 5.0 & 4.8 \\
\hline Private & 17.9 & 18.7 & 17.3 & 18.7 & 18.8 & 14.5 \\
\hline Gross national saving & 18.1 & 19.0 & 17.5 & 18.5 & 18.8 & 21.1 \\
\hline Public & 4.4 & 3.1 & 3.1 & 4.5 & 4.3 & 1.5 \\
\hline Private & 13.7 & 15.9 & 14.4 & 14.0 & 14.4 & 19.6 \\
\hline Foreign saving & 5.5 & 4.5 & 4.3 & 4.6 & 5.1 & -1.9 \\
\hline Public saving-investment gap & -1.3 & -1.7 & -1.4 & 0.0 & -0.7 & -3.3 \\
\hline Private saving-investment gap & -4.2 & -2.8 & -2.9 & -4.6 & -4.4 & 5.2 \\
\hline
\end{tabular}

Source: Data provided by the Philippine authorities. 
Table 6. Philippines: Price Developments, 1993-98

(Annual percentage changes)

\begin{tabular}{|c|c|c|c|c|c|c|c|}
\hline & Weights & 1993 & 1994 & 1995 & $\cdot 1996$ & 1997 & 1998 \\
\hline \multicolumn{8}{|l|}{ Consumer prices $1 /$} \\
\hline End of period (all items) & $\ldots$ & 8.4 & 7.2 & 10.9 & 5.2 & 7.3 & 10.5 \\
\hline Period average (all items) & 100.0 & 7.6 & 9.0 & 8.1 & 8.4 & 5.1 & 9.0 \\
\hline Food, beverages, and tobacco & 58.5 & 6.1 & 8.3 & 9.5 & 10.2 & 1.9 & 7.7 \\
\hline Clothing & 4.4 & 7.4 & 4.8 & 3.3 & 3.6 & 3.8 & 5.7 \\
\hline Housing and repairs & 13.3 & 12.6 & 13.1 & 10.7 & 9.6 & 9.7 & 10.6 \\
\hline Fuel, light, and water & 5.4 & 7.3 & 6.9 & 3.0 & 6.6 & 8.4 & 7.6 \\
\hline Services & 10.8 & 7.8 & 8.9 & 6.5 & 9.5 & 13.2 & 14.3 \\
\hline Miscellaneous & 7.6 & 8.4 & 11.0 & 0.9 & -3.8 & 2.0 & 7.1 \\
\hline Wholesale prices $2 /$ & $\ldots$ & -1.1 & 8.2 & 3.2 & 9.1 & $0.53 /$ & 11.7 \\
\hline GNP deflator 3/ & $\ldots$ & 5.1 & 10.0 & 7.4 & 7.9 & 6.0 & 10.4 \\
\hline
\end{tabular}

Source: Data provided by the Philippine authorities.

1/ For the Philippines as a whole.

2/ Period averages for Metro Manila.

3/ Period averages. 
Table 7. Philippines: Labor Market Developments, 1993-98

\begin{tabular}{|c|c|c|c|c|c|c|}
\hline & 1993 & 1994 & 1995 & 1996 & 1997 & 1998 \\
\hline Population & 65,650 & 67,040 & 68,350 & 69,952 & 71,550 & 73,148 \\
\hline Total labor force & 26,879 & 27,654 & 28,382 & 29,733 & 30,355 & 31,056 \\
\hline Unemployed & 2,497 & 2,622 & 2,704 & 2,546 & 2,640 & 3,144 \\
\hline Unemployment rate (percent) & 9 & 10 & 10 & 9 & 9 & 10 \\
\hline Total employed & 24,382 & 25,032 & 25,676 & 27,186 & 27,715 & 27,911 \\
\hline Agriculture & 11,139 & 11,286 & 11,147 & 11,645 & 11,314 & 10,933 \\
\hline Industry & 3,804 & 3,948 & 4,139 & 4,430 & 4,631 & 4,583 \\
\hline Mining and quarrying & 135 & 111 & 107 & 113 & 130 & 120 \\
\hline Manufacturing & 2,457 & 2,539 & 2,617 & 2,696 & 2,732 & 2,716 \\
\hline Construction & 1,110 & 1,187 & 1,302 & 1,504 & 1,637 & 1,605 \\
\hline Utilities & 102 & 112 & 114 & 118 & 132 & 143 \\
\hline Services & 9,439 & 9,798 & 10,391 & 11,112 & 11,771 & 12,395 \\
\hline Transportation and communications & 1,291 & 1,393 & 1,477 & 1,630 & 1,742 & 1,849 \\
\hline Trade & 3,389 & 3,520 & 3,767 & 4,013 & 4,138 & 4,312 \\
\hline Finance & 503 & 490 & 535 & 614 & 688 & 673 \\
\hline Government services & 4,244 & 4,386 & 4,600 & 4,850 & 5,196 & 5,555 \\
\hline Other & 13 & 10 & 12 & 4 & 6 & 6 \\
\hline Total employed & 100.0 & 100.0 & 100.0 & 100.0 & 100.0 & 100.0 \\
\hline Agriculture & 45.7 & 45.1 & 43.4 & 42.8 & 40.8 & 39.2 \\
\hline Industry & 15.6 & 15.8 & 16.1 & 16.3 & 16.7 & 16.4 \\
\hline Mining and quarrying & 0.6 & 0.4 & 0.4 & 0.4 & 0.5 & 0.4 \\
\hline Manufacturing & 10.1 & 10.1 & 10.2 & 9.9 & 9.9 & 9.7 \\
\hline Construction & 4.6 & 4.7 & 5.1 & 5.5 & 5.9 & 5.8 \\
\hline Utilities & 0.4 & 0.4 & 0.4 & 0.4 & 0.5 & 0.5 \\
\hline Services & 38.7 & 39.1 & 40.5 & 40.9 & 42.5 & 44.4 \\
\hline Transportation and communications & 5.3 & 5.6 & 5.8 & 6.0 & 6.3 & 6.6 \\
\hline Trade & 13.9 & 14.1 & 14.7 & 14.8 & 14.9 & 15.4 \\
\hline Finance & 2.1 & 2.0 & 2.1 & 2.3 & 2.5 & 2.4 \\
\hline Government services & 17.4 & 17.5 & 17.9 & 17.8 & 18.7 & 19.9 \\
\hline Other & 0.1 & 0.0 & 0.0 & 0.0 & 0.0 & 0.0 \\
\hline
\end{tabular}

Source: Data provided by the Philippine authorities. 
Table 8. Philippines: Wages and Productivity, 1993-98

\begin{tabular}{|c|c|c|c|c|c|c|}
\hline & 1993 & 1994 & 1995 & 1996 & 1997 & 1998 \\
\hline \multicolumn{7}{|l|}{ Minimum wages } \\
\hline \multicolumn{7}{|l|}{ Agriculture } \\
\hline \multicolumn{7}{|l|}{ Plantation } \\
\hline National capital region & 108.0 & 135.0 & 135.0 & 155.0 & 175.0 & 186 \\
\hline Outside national capital region & $79.0-102.0$ & $87.0-128.0$ & $76.0-128.0$ & $93.8-145.0$ & $94.4-150.0$ & $104.4-163.0$ \\
\hline \multicolumn{7}{|l|}{ Nonplantation } \\
\hline National capital region & 97.5 & 124.5 & 124.5 & 144.5 & 164.0 & 179.5 \\
\hline Outside national capital region & $58.5-82.5$ & $62.1-107.5$ & $62.1-107.5$ & $76.5-125.0$ & $79.9-130.0$ & $89.85-143.0$ \\
\hline \multicolumn{7}{|l|}{ Nonagriculture } \\
\hline Nationa capital region & 119.4 & 145.0 & 145.0 & 165.0 & 185.0 & 185 \\
\hline Outside national capital region & $89.0-127.0$ & $93.0-138.0$ & $92.1-138.0$ & $94.0-155.0$ & $101.0-175.0$ & $111.0-188.0$ \\
\hline \multicolumn{7}{|l|}{ Minimum wages (real growth) } \\
\hline \multicolumn{7}{|l|}{ Agriculture } \\
\hline \multicolumn{7}{|l|}{ Plantation } \\
\hline National capital region & -8.5 & 14.5 & -7.6 & 5.9 & 5.9 & -3.58 \\
\hline Outside national capital region & $(-5.51)-(-13.95)$ & $(1.87)-(16.08)$ & $(-19.06)-(-7.41)$ & $(12.89)-(3.68)$ & $(-4.69)-(-2.03)$ & $(0.95)-(-0.81)$ \\
\hline \multicolumn{7}{|l|}{ Nonplantation } \\
\hline National capital region & -8.5 & 17.0 & -7.6 & 7.1 & 6.4 & -0.71 \\
\hline Outside national capital region & $(-5.51)-(-5.51)$ & $(-1.18)-(20.53)$ & $(-8.0)-(-7.41)$ & $(12.75)-(6.42)$ & $(-1.15) \cdot(-1.51)$ & $(2.71)-(0.41)$ \\
\hline \multicolumn{7}{|l|}{ Nonagriculture } \\
\hline National capital region & -7.4 & 11.2 & -7.6 & 5.0 & 5.1 & -2.91 \\
\hline Outside national capital region & $(-5.51)-(6.19)$ & $(-3.34)-(0.51)$ & $(-8.2)-(-7.41)$ & $(-6.69)-(2.8)$ & $(1.76)-(6.92)$ & $(0.32)-(-1.94)$ \\
\hline \multicolumn{7}{|l|}{ Productivity (real growth) } \\
\hline All sectors & -0.8 & 1.7 & 2.1 & 0.0 & 3.2 & -1.2 \\
\hline Agriculture & -1.7 & 1.3 & 2.1 & -0.6 & 6.0 & -3.4 \\
\hline Industry & 2.2 & 1.9 & 1.8 & -0.6 & 1.6 & -0.7 \\
\hline Of which: Manufacturing & 3.5 & 1.6 & 3.6 & 2.5 & 2.9 & -0.5 \\
\hline Services & -0.7 & 0.4 & -1.0 & -0.5 & -0.4 & -1.7 \\
\hline
\end{tabular}

Source: Data provided by the Philippine authorities.

CInternational Monetary Fund. Not for Redistribution 
Table 9. Philippines: Domestic Retail Prices of Petroleum Products, 1993-98

(In pesos per liter; end of period)

\begin{tabular}{lrrrrrr}
\hline & 1993 & 1994 & 1995 & 1996 & 1997 & 1998 \\
\hline Unleaded Gasoline & $\ldots$ & 9.00 & 9.00 & 10.73 & 11.58 & 11.39 \\
Regular gasoline & 9.50 & 8.50 & 8.50 & 9.99 & 10.63 & 9.96 \\
Premium gasoline & 10.00 & 9.00 & 9.00 & 10.74 & 12.10 & 11.76 \\
Diesel oil & 7.00 & 7.00 & 7.00 & 8.07 & 7.83 & 8.05 \\
Kerosene & 7.00 & 7.00 & 7.00 & 7.27 & 6.94 & 6.63 \\
Aviation fuel & 13.39 & 13.39 & 13.39 & 9.83 & 9.90 & 9.58 \\
LPG & 5.26 & 5.26 & 5.26 & 6.70 & 7.11 & 6.84 \\
Fuel oil & 2.65 & 2.65 & 2.65 & 4.17 & 4.52 & 4.89 \\
Weighted average & 5.66 & 5.42 & 5.26 & 6.97 & 7.17 & 7.40 \\
\hline
\end{tabular}

Source: Department of Energy. 
Table 10. Philippines: Consolidated Public Sector Balance, 1993-98

\begin{tabular}{|c|c|c|c|c|c|c|}
\hline & 1993 & 1994 & 1995 & 1996 & 1997 & 1998 \\
\hline & \multicolumn{6}{|c|}{ (ln billions of pesos) } \\
\hline Consolidated public sector balance & -25.9 & -8.7 & -4.1 & 4.6 & -23.3 & -84.3 \\
\hline \multicolumn{7}{|l|}{ Monitored public sector } \\
\hline borrowing requirement (PSBR) & -55.8 & -7.5 & -16.7 & -12.4 & -40.3 & -116.4 \\
\hline National government balance & -21.9 & 0.0 & 11.1 & 6.3 & 1.6 & -50.0 \\
\hline CB-BOL net income & -15.1 & -8.1 & -20.0 & -13.8 & -25.7 & -26.4 \\
\hline Monitored corporations' balance & -25.6 & -9.7 & -1.3 & -11.2 & -17.9 & -41.7 \\
\hline OPSF balance & -7.9 & 2.6 & -9.2 & 4.8 & -0.8 & 0.7 \\
\hline Adjustments for intra-PSBR transfers & 14.7 & 7.6 & 2.8 & 1.5 & 2.5 & 0.9 \\
\hline \multicolumn{7}{|l|}{ Borrowing requirement of remaining } \\
\hline public sector & 29.9 & -1.2 & 12.6 & 17.0 & 17.0 & 32.1 \\
\hline \multicolumn{7}{|l|}{ Surplus of government financial } \\
\hline institutions (GFIs) & 6.1 & 3.] & 5.0 & 8.4 & 4.4 & 5.4 \\
\hline \multicolumn{7}{|l|}{$\begin{array}{l}\text { Net income of the Central Bank of } \\
\text { the Philippines }\end{array}$} \\
\hline Net income of BSP & -1.0 & 5.3 & 3.6 & -2.3 & 2.2 & 7.2 \\
\hline Local government & 5.8 & 4.6 & 2.0 & 3.0 & 4.2 & 2.0 \\
\hline Social security & 11.7 & -12.0 & 0.0 & 8.5 & 3.9 & 17.7 \\
\hline \multicolumn{7}{|l|}{ Adjustment for transfers within the } \\
\hline \multirow[t]{2}{*}{ public sector } & 7.2 & -2.2 & 2.0 & -0.6 & 2.3 & -0.3 \\
\hline & \multicolumn{6}{|c|}{ (In percent of GNP) } \\
\hline Consolidated public sector balance & $-1.7 \%$ & $-0.5 \%$ & $-0.2 \%$ & $0.2 \%$ & $-0.9 \%$ & $-3.0 \%$ \\
\hline \multicolumn{7}{|l|}{ Monitored public sector borrowing } \\
\hline requirement (PSBR) & $-3.7 \%$ & $-0.4 \%$ & $.0 .9 \%$ & $-0.5 \%$ & $-1.6 \%$ & $-4.2 \%$ \\
\hline National government balance & $-1.5 \%$ & $0.9 \%$ & $0.6 \%$ & $0.3 \%$ & $0.1 \%$ & $-1.8 \%$ \\
\hline Monitored corporations' balance & $-1.7 \%$ & $-0.6 \%$ & $-0.1 \%$ & $-0.5 \%$ & $-0.7 \%$ & $-1.5 \%$ \\
\hline \multicolumn{7}{|l|}{ Underlying consolidated public sector } \\
\hline balance & $-2.1 \%$ & $-2.4 \%$ & $-1.5 \%$ & $-0.1 \%$ & $-1.5 \%$ & $-3.1 \%$ \\
\hline \multicolumn{7}{|l|}{ Memorandum item: } \\
\hline GNP (in billion of pesos) & 1,500 & 1,736 & 1,959 & 2,261 & 2,523 & 2,794 \\
\hline
\end{tabular}


Table 11. Philippines: Operations of the National Government, 1993-98

(In billions of pesos)

\begin{tabular}{|c|c|c|c|c|c|c|}
\hline & 1993 & 1994 & 1995 & 1996 & 1997 & 1998 \\
\hline Revenue and grants & 260.4 & 348.9 & 374.6 & 431.7 & 493.4 & 472.7 \\
\hline Tax revenue & 230.2 & 271.3 & 310.5 & 367.9 & 412.2 & 416.6 \\
\hline Domestic & 147.5 & 189.0 & 212.6 & 263.0 & 317.0 & 340.2 \\
\hline Income and profits & 74.8 & 91.9 & 111.2 & 136.4 & 164.2 & 183.9 \\
\hline Excise & 30.3 & 39.6 & 38.5 & 48.4 & 63.0 & 60.9 \\
\hline Sales tax/license $1 /$ & 33.5 & 36.5 & 46.3 & 59.3 & 67.7 & 67.9 \\
\hline Other domestic taxes & 9.0 & 21.1 & 16.5 & 19.0 & 22.1 & 27.6 \\
\hline International trade taxes & 82.7 & 82.3 & 98.0 & 104.9 & 95.2 & 76.4 \\
\hline Import duties and taxes $2 /$ & 82.0 & 81.6 & 97.6 & 104.6 & 94.8 & 76.0 \\
\hline Others $3 /$ & 0.7 & 0.7 & 0.4 & 0.3 & 0.4 & 0.4 \\
\hline Nontax revenue & 30.2 & 77.6 & 64.1 & 63.8 & 81.2 & 56.1 \\
\hline Domestic & 28.2 & 76.8 & 63.1 & 63.3 & 79.5 & 55.7 \\
\hline Other offices & 21.1 & 26.5 & 25.4 & 35.1 & 47.8 & 42.5 \\
\hline Sequestered assets & 0.1 & 15.3 & 1.3 & 0.3 & 2.7 & 1.22 \\
\hline Sales of assets & 1.6 & 14.6 & 21.6 & 5.4 & 6.7 & 0.5 \\
\hline Interest on central bank deposits & 4.8 & 5.5 & 1.2 & 0.8 & 0.5 & 0.5 \\
\hline $\mathrm{CB}-\mathrm{BOL}$ interest, rebate, and dividend & $\ldots$ & 12.7 & 13.4 & 21.3 & 21.5 & 10.1 \\
\hline \multicolumn{7}{|l|}{ Interest from nonfinancial } \\
\hline government corporations & 0.6 & 2.2 & 0.3 & 0.5 & 0.2 & 0.8 \\
\hline ESAF & 0.5 & 0.0 & 0.0 & 0.0 & 0.0 & 0.0 \\
\hline Foreign grants & 1.5 & 0.7 & 1.0 & 0.6 & 1.7 & 0.4 \\
\hline Domestic grants & 0.0 & 0.0 & 0.0 & 0.0 & 0.0 & 0.0 \\
\hline Expenditure & 282.3 & 348.8 & 378.7 & 439.3 & 502.9 & 544.2 \\
\hline Current expenditure & 226.6 & 296.4 & 305.8 & 363.6 & 403.7 & 456.1 \\
\hline Personnel services & 78.7 & 92.7 & 109.1 & 135.4 & 172.8 & 198.5 \\
\hline Maintenance and operations & 34.6 & 46.8 & 47.0 & 48.7 & 51.9 & 64.4 \\
\hline Interest payments & 76.5 & 108.1 & 101.1 & 111.6 & 110.6 & 131.5 \\
\hline Domestic & 56.2 & 88.8 & 79.2 & 94.1 & 91.0 & 105.2 \\
\hline Of which: $\mathrm{CB}-\mathrm{BOL}$ restructuring external & $\ldots$ & 29.0 & 28.4 & 35.1 & 32.6 & 31.7 \\
\hline Foreign & 20.3 & 19.3 & 21.9 & 17.5 & 19.6 & 26.3 \\
\hline Subsidies 4/ & 9.1 & 11.4 & 7.2 & 22.6 & 12.0 & 4.9 \\
\hline Of which: Tax expenditures & 3.9 & 4.4 & 3.6 & 6.7 & 6.7 & 0.2 \\
\hline Allotments to LGUs & 27.8 & 37.4 & 41.4 & 45.3 & 56.4 & 56.9 \\
\hline Capital expenditure and net lending & 55.7 & 52.4 & 72.9 & 75.7 & 99.2 & 88.0 \\
\hline Capital expenditures & 44.8 & 43.4 & 64.5 & 69.6 & 93.6 & 86.5 \\
\hline Infrastructure and other capital outlays & 37.8 & 33.6 & 52.7 & 57.5 & 79.1 & 71.3 \\
\hline Transfers to local government units & 6.9 & 9.8 & 11.8 & 12.1 & 14.5 & 15.1 \\
\hline Equity and net lending & 9.4 & 9.0 & 8.4 & 3.2 & 3.0 & 1.6 \\
\hline $\mathrm{CARP}$ land acquisition and credit & 1.5 & 0.0 & 0.0 & 2.9 & 2.6 & 0.5 \\
\hline Deficit $(-)$ & $(21.9)$ & 0.1 & (4.1) & $(7.6)$ & $(9.5)$ & $(71.5)$ \\
\hline Excluding CB restructuring & $(21.9)$ & 16.3 & 11.1 & 6.3 & 1.6 & $(50.0)$ \\
\hline Financing & 21.9 & $(16.3)$ & $(11.1)$ & $(6.3)$ & $(1.6)$ & 50.0 \\
\hline Foreign (net) & 12.9 & $(11.6)$ & $(13.3)$ & $(5.9)$ & $(6.8)$ & 12.4 \\
\hline Domestic (net) & 9.0 & $(4.7)$ & 2.3 & $(0.4)$ & 5.2 & 37.6 \\
\hline
\end{tabular}

Sources: Data provided by the Philippine authorities; and Fund staff estimates.

$1 /$ Including domestic VAT

2/ Including VAT on imports.

3/ Including travel tax.

4/ Including transfer to the OPSF and tax expenditures. 
Table 12. Philippines: National Government Revenues and Grants, 1993-98

\begin{tabular}{|c|c|c|c|c|c|c|}
\hline & 1993 & 1994 & 1995 & 1996 & 1997 & 1998 \\
\hline & \multicolumn{6}{|c|}{ (In billions of pesos) } \\
\hline Total revenue and grants & 260.4 & 348.9 & 374.7 & 431.7 & 493.4 & 472.7 \\
\hline Tax revenue & 230.2 & 271.3 & 310.5 & 367.9 & 412.2 & 416.6 \\
\hline Taxes on net income and profits & 74.8 & 91.9 & 111.2 & 136.4 & 164.2 & 183.9 \\
\hline Taxes on domestic production and consumption & 63.7 & 76.0 & 84.8 & 107.7 & 130.7 & 128.7 \\
\hline Sales and business taxes & 33.5 & 36.5 & 46.3 & 59.3 & 67.7 & 67.9 \\
\hline Of which : VAT & 22.8 & 25.5 & 28.8 & 40.9 & 47.3 & 47.5 \\
\hline Excises $1 /$ & 30.3 & 39.6 & 38.5 & 48.4 & 63.0 & 60.9 \\
\hline Tobacco products & 10.3 & 11.7 & 13.8 & 15.0 & 15.9 & 17.1 \\
\hline Alcoholic beverages & 8.2 & 9.7 & 10.1 & 11.1 & 13.4 & 14.3 \\
\hline Petroleum products & 8.7 & 10.6 & 10.1 & 16.1 & 29.3 & 27.1 \\
\hline Taxes on international trade and transactions & 82.7 & 82.3 & 98.0 & 104.9 & 95.2 & 76.4 \\
\hline Import duties and import taxes & 82.0 & 81.6 & 97.6 & 104.6 & 94.8 & 76.0 \\
\hline Others $2 /$ & 0.7 & 0.7 & 0.4 & 0.3 & 0.4 & 0.4 \\
\hline Other domestic taxes 3/ & 9.0 & 21.1 & 16.5 & 19.0 & 22.1 & 27.6 \\
\hline \multirow[t]{2}{*}{ Nontax revenue $4 /$} & 30.2 & 77.6 & 64.1 & 63.8 & 81.2 & 56.1 \\
\hline & \multicolumn{6}{|c|}{ (In percent of total revenues) } \\
\hline Total revenue and grants & 100.0 & 100.0 & 100.0 & 100.0 & 100.0 & 100.0 \\
\hline Tax revenue & 88.4 & 77.8 & 82.9 & 85.2 & 83.5 & 88.1 \\
\hline Taxes on net income and profits & 28.7 & 26.3 & 29.7 & 31.6 & 33.3 & 38.9 \\
\hline Taxes on domestic production and consumption & 24.5 & 21.8 & 22.6 & 24.9 & 26.5 & 27.2 \\
\hline Sales and business taxes & 12.9 & 10.5 & 12.4 & 13.7 & 13.7 & 14.4 \\
\hline Of which : VAT & 8.8 & 7.3 & 7.7 & 9.5 & 9.6 & 10.0 \\
\hline Excises 1/ & 11.6 & 11.3 & 10.3 & 11.2 & 12.8 & 12.9 \\
\hline Tobacco products & 4.0 & 3.4 & 3.7 & 3.5 & 3.2 & 3.6 \\
\hline Alcoholic beverages & 3.1 & 2.8 & 2.7 & 2.6 & 2.7 & 3.0 \\
\hline Petroleum products & 3.3 & 3.0 & 2.7 & 3.7 & 5.9 & 5.7 \\
\hline Taxes on international trade and transactions & 31.8 & 23.6 & 26.2 & 24.3 & 19.3 & 16.2 \\
\hline Import duties and import taxes & 31.5 & 23.4 & 26.0 & 24.2 & 19.2 & 16.1 \\
\hline Others $2 I$ & 0.3 & 0.2 & 0.1 & 0.1 & 0.1 & 0.1 \\
\hline Other domestic taxes $3 /$ & 3.5 & 6.0 & 4.4 & 4.4 & 4.5 & 5.8 \\
\hline \multirow[t]{2}{*}{ Nontax revenue $4 /$} & 11.6 & 22.2 & 17.1 & 14.8 & 16.5 & 11.9 \\
\hline & \multicolumn{6}{|c|}{ (In percent of GNP) } \\
\hline Total revenue and grants & 17.4 & 20.1 & 19.1 & 19.1 & 19.6 & 16.9 \\
\hline Tax revenue & 15.3 & 15.6 & 15.9 & 16.3 & 16.3 & 14.9 \\
\hline Taxes on net income and profits & 5.0 & 5.3 & 5.7 & 6.0 & 6.5 & 6.6 \\
\hline Taxes on domestic production and consumption & 4.2 & 4.4 & 4.3 & 4.8 & 5.2 & 4.6 \\
\hline Sales and business taxes & 2.2 & 2.1 & 2.4 & 2.6 & 2.7 & 2.4 \\
\hline Of which: VAT & 1.8 & 2.0 & 1.5 & 1.8 & 1.9 & 1.7 \\
\hline Excises $1 /$ & 2.0 & 2.3 & 2.0 & 2.1 & 2.5 & 2.2 \\
\hline Tobacco products & 0.7 & 0.7 & 0.7 & 0.7 & 0.6 & 0.6 \\
\hline Alcoholic beverages & 0.5 & 0.6 & 0.5 & 0.5 & 0.5 & 0.5 \\
\hline Petroleum products & 0.6 & 0.6 & 0.5 & 0.7 & 1.2 & 1.0 \\
\hline Taxes on international trade and transactions & 5.5 & 4.7 & 5.0 & 4.6 & 3.8 & 2.7 \\
\hline Import duties and import taxes & 5.5 & 4.7 & 5.0 & 4.6 & 3.8 & 2.7 \\
\hline Others $2 /$ & 0.0 & 0.0 & 0.0 & 0.0 & 0.0 & 0.0 \\
\hline Other domestic taxes $3 /$ & 0.6 & 1.2 & 0.8 & 0.8 & 0.9 & 1.0 \\
\hline Nontax revenue $4 /$ & 2.0 & 4.5 & 3.3 & 2.8 & 3.2 & 2.0 \\
\hline
\end{tabular}

Source: Data provided by the Philippine authorities.

1/ Data on the components of excise duties are based on BIR collections, which differ slightly from the records of the Treasury. 2/ Foreign exchange tax and travel tax.

$3 /$ Including property taxes.

4/ Including grants and Economic Support Fund (ESF) and CB restructuring. 
Table 13. Philippines: National Government Expenditures and Net Lending, 1993-98

\begin{tabular}{|c|c|c|c|c|c|c|}
\hline & 1993 & 1994 & 1995 & 1996 & 1997 & 1998 \\
\hline & \multicolumn{6}{|c|}{ (In billions of pesos) } \\
\hline Total expenditure and net lending & 282.3 & 348.8 & 378.7 & 439.3 & 502.9 & 544.2 \\
\hline Current expenditure & 226.6 & 296.4 & 305.8 & 363.6 & 403.7 & 456.1 \\
\hline Personnel services & 78.7 & 92.7 & 109.1 & 135.4 & 172.8 & 198.5 \\
\hline Maintenance and other operational expens & 34.6 & 46.8 & 47.0 & 65.4 & 58.0 & 63.4 \\
\hline Interest payments & 76.5 & 108.1 & 101.1 & 111.6 & 110.6 & 131.5 \\
\hline Allotments to local government units & 27.8 & 37.4 & 41.4 & 45.3 & 56.4 & 56.9 \\
\hline Subsidies & 9.1 & 11.5 & 7.2 & 5.9 & 5.9 & 5.9 \\
\hline Capital expenditure & 44.8 & 43.4 & 64.5 & 69.6 & 93.6 & 86.5 \\
\hline Infrastructure and other capital outlays & 37.9 & 33.6 & 52.7 & 57.5 & 79.1 & 71.3 \\
\hline Transfers to LGUs & 6.9 & 9.8 & 11.8 & 12.1 & 14.5 & 15.1 \\
\hline Equity and net lending & 10.9 & 9.0 & 8.4 & 6.1 & 5.6 & 1.6 \\
\hline Equity $1 /$ & 6.8 & 3.1 & 4.6 & 4.9 & 4.2 & 1.3 \\
\hline \multirow[t]{2}{*}{ Loans less repayments } & 4.2 & 5.9 & 3.8 & 1.2 & 1.4 & 0.3 \\
\hline & \multicolumn{6}{|c|}{ (In percent of total expenditure and net lending) } \\
\hline Total expenditure and net lending & 100.0 & 100.0 & 100.0 & 100.0 & 100.0 & 100.0 \\
\hline Current expenditure & 80.3 & 85.0 & 80.7 & 82.8 & 80.3 & 83.8 \\
\hline Personnel services & 27.9 & 26.6 & 28.8 & 30.8 & 34.4 & 36.5 \\
\hline Maintenance and other operational expens & 12.3 & 13.4 & 12.4 & 14.9 & 11.5 & 11.6 \\
\hline Interest payments & 27.1 & 31.0 & 26.7 & 25.4 & 22.0 & 24.2 \\
\hline Allotments to local government units & 9.8 & 10.7 & 10.9 & 10.3 & 11.2 & 10.5 \\
\hline Subsidies & 3.2 & 3.3 & 1.9 & 1.3 & 1.2 & 1.1 \\
\hline Capital expenditure & 15.9 & 12.4 & 17.0 & 15.8 & 18.6 & 15.9 \\
\hline Infrastructure and other capital outlays & 13.4 & 9.6 & 13.9 & 13.1 & 15.7 & 13.1 \\
\hline Transfers to LGUs & 2.4 & 2.8 & 3.1 & 2.8 & 2.9 & 2.8 \\
\hline Equity and net lending & 3.9 & 2.6 & 2.2 & 1.4 & 1.1 & 0.3 \\
\hline Equity $\mathbf{l}$ & 2.4 & 0.9 & 1.2 & 1.1 & 0.8 & 0.2 \\
\hline \multirow[t]{2}{*}{ Loans less repayments } & 1.5 & 1.7 & 1.0 & 0.3 & 0.3 & 0.1 \\
\hline & \multicolumn{6}{|c|}{ (In percent of GNP) } \\
\hline Total expenditure and net lending & 18.8 & 20.1 & 19.2 & 19.4 & 19.9 & 19.5 \\
\hline Current expenditure & 15.1 & 17.1 & 15.5 & 16.1 & 16.0 & 16.3 \\
\hline Personnel services & 5.2 & 5.3 & 5.5 & 6.0 & 6.8 & 7.1 \\
\hline Maintenance and other operational expens & 2.3 & 2.7 & 2.4 & 2.9 & 2.3 & 2.3 \\
\hline Interest payments & 5.1 & 6.2 & 5.1 & 4.9 & 4.4 & 4.7 \\
\hline Allotments to local government units & 1.9 & 2.2 & 2.1 & 2.0 & 2.2 & 2.0 \\
\hline Subsidies & 0.6 & 0.7 & 0.4 & 0.3 & 0.2 & 0.2 \\
\hline Capital expenditure & 3.0 & 2.5 & 3.3 & 3.1 & 3.7 & 3.1 \\
\hline Infrastructure and other capital outlays & 2.5 & 1.9 & 2.7 & 2.5 & 3.1 & 2.6 \\
\hline Transfers to LGUs & 0.5 & 0.6 & 0.6 & 0.5 & 0.6 & 0.5 \\
\hline Equity and net lending & 0.7 & 0.5 & 0.4 & 0.3 & 0.2 & 0.1 \\
\hline Equity $1 /$ & 0.5 & 0.2 & 0.2 & 0.2 & 0.2 & 0.0 \\
\hline Loans less repayments & 0.3 & 0.3 & 0.2 & 0.1 & 0.1 & 0.0 \\
\hline
\end{tabular}

Source: Data provided by the Philippine authorities.

1/ Including CARP land acquisition and credit. 
Table 14. Philippines: Operations of Social Security Institutions, 1993-98 1/

(In billions of pesos)

\begin{tabular}{|c|c|c|c|c|c|c|}
\hline & 1993 & 1994 & 1995 & 1996 & 1997 & 1998 \\
\hline Revenues & 43.7 & 45.8 & 49.1 & 54.2 & 63.6 & 84.6 \\
\hline Contributions $2 /$ & 23.7 & 26.5 & 31.5 & 34.7 & 36.5 & 57.8 \\
\hline Of which: National government $3 /$ & 4.6 & 3.5 & 5.4 & 4.8 & 2.8 & 13.3 \\
\hline Other revenues & 20.1 & 19.2 & 17.6 & 19.5 & 27.1 & 26.8 \\
\hline Of which: National government securities & 12.2 & 12.4 & 9.2 & 7.9 & 9.0 & 12.8 \\
\hline Expenditures & 39.2 & 50.5 & 50.7 & 52.7 & 64.0 & 80.3 \\
\hline Social security benefits & 21.3 & 24.5 & 26.3 & 29.0 & 30.7 & 40.2 \\
\hline Operating and other expenditures & 3.5 & 11.0 & 6.3 & 7.8 & 6.8 & 12.1 \\
\hline Net lending & 14.3 & 15.0 & 18.1 & 15.9 & 26.5 & 28.0 \\
\hline Of which: National government & 7.1 & -7.2 & 1.6 & 6.9 & 4.3 & 13.5 \\
\hline Surplus/deficit & 4.6 & -4.8 & -1.6 & 1.6 & -0.5 & 4.2 \\
\hline \multicolumn{7}{|l|}{ Surplus/deficit before net } \\
\hline lending to national government & 11.7 & -12.0 & 0.0 & 8.5 & 3.8 & 17.7 \\
\hline
\end{tabular}

Source: Data provided by the Philippine authorities.

1/ Social Security System (SSS) and Government Service Insurance System (GSIS) including Philippine Health Insurance Corporation (PHIC) starting 1997.

2/ By employees and employers.

3/ National government's contribution, as employer, to the GSIS. 
Table 15. Philippines: Local Governments Budgetary Operations, 1993-98

(In billions of pesos)

\begin{tabular}{|c|c|c|c|c|c|c|}
\hline & 1993 & 1994 & 1995 & 1996 & $19971 /$ & $19981 /$ \\
\hline Revenue & 44.3 & 57.6 & 66.9 & 75.4 & 96.7 & 1.06 .9 \\
\hline Local source revenue & 16.3 & 21.7 & 26.1 & 28.7 & 37.4 & 46.7 \\
\hline Property taxes & 4.9 & 4.2 & 7.3 & 9.9 & 10.4 & 12.8 \\
\hline Business taxes & 5.3 & 8.8 & 8.4 & 8.7 & 12.5 & 15.8 \\
\hline Nontax revenue & 6.1 & 8.7 & 10.4 & 10.1 & 14.5 & 18.1 \\
\hline Grants and aid & 28.0 & 35.9 & 40.8 & 46.7 & 59.3 & 60.2 \\
\hline Statutory allotments & 27.5 & 35.2 & 40.1 & 45.9 & 58.5 & 59.5 \\
\hline Special aid from national government & 0.5 & 0.7 & 0.7 & 0.8 & 0.8 & 0.7 \\
\hline Expenditure & 38.5 & 53.0 & 64.9 & 72.4 & 92.5 & 104.9 \\
\hline Current & 34.1 & 46.1 & 56.5 & 64.1 & 83.0 & 92.0 \\
\hline Of which : economic development & 5.3 & 7.4 & 9.1 & 9.7 & 11.6 & 14.2 \\
\hline Capital outlays & 4.4 & 6.9 & 8.4 & 8.3 & 9.5 & 12.9 \\
\hline Surplus/deficit & 5.8 & 4.6 & 2.0 & 3.0 & 4.2 & 2.0 \\
\hline
\end{tabular}

Source: Data provided by Philippine authorities.

1/ Preliminary actual. 
Table 16. Philippines: Public Sector Infrastructure Program, 1993-98

(In billions of pesos)

\begin{tabular}{|c|c|c|c|c|c|c|}
\hline & 1993 & $19941 /$ & $19951 /$ & $19961 /$ & $19971 /$ & $19982 /$ \\
\hline Transportation & 10.4 & 19.7 & 21.2 & 26.3 & 31.8 & 34.6 \\
\hline Highways & 7.4 & 14.3 & 13.6 & 21.9 & 21.8 & 21.2 \\
\hline Railways & 0.2 & 0.3 & 0.6 & 0.6 & 0.6 & 0.2 \\
\hline Airports and airways & 1.1 & 2.1 & 5.1 & 2.2 & 2.9 & 7.6 \\
\hline Ports/shore protection/lighthouses & 1.6 & 2.7 & 1.6 & 1.1 & 3.1 & 2.4 \\
\hline Mass transit & 0.1 & 0.3 & 0.3 & 0.5 & 3.4 & 3.2 \\
\hline Communications & 1.0 & 1.8 & 1.1 & 1.1 & 2.0 & 2.2 \\
\hline Water resources & 5.4 & 5.9 & 7.0 & 13.0 & 8.9 & 9.0 \\
\hline Irrigation & 2.4 & 2.7 & 2.6 & 3.3 & 2.1 & 3.5 \\
\hline Water supply & 2.0 & 1.2 & 1.8 & 5.2 & 3.7 & 1.9 \\
\hline Food control and drainage & 1.0 & 2.0 & 2.6 & 4.5 & 3.1 & 3.6 \\
\hline Power and rural electrification & 31.0 & 39.0 & 20.0 & 20.9 & 28.2 & 22.3 \\
\hline Power & 26.4 & 35.3 & 16.8 & 20.0 & 27.1 & 21.4 \\
\hline Rural electrification & 0.6 & 0.1 & 0.0 & 0.9 & 1.1 & 0.9 \\
\hline Other & 4.0 & 3.6 & 3.2 & 0.0 & 0.0 & 0.0 \\
\hline Social infrastructure & 1.8 & 30.8 & 42.6 & 28.8 & 31.1 & 30.0 \\
\hline School buildings & 1.0 & 3.3 & 5.0 & 5.1 & 4.5 & 3.1 \\
\hline Related infrastructure & 0.8 & 27.5 & 37.6 & 23.7 & 26.6 & 26.9 \\
\hline Total & 49.6 & 97.2 & 91.9 & 90.1 & 102.0 & 98.1 \\
\hline
\end{tabular}

Source: Data provided by the Philippine authorities.

1/ Figures calculated on an obligations basis.

2/ Preliminary actual. 
Table 17. Philippines: Statement of Financial Operations of

14 Major Monitored Public Corporations, 1993-98

(In billions of pesos)

\begin{tabular}{|c|c|c|c|c|c|c|}
\hline & 1993 & 1994 & 1995 & 1996 & 1997 & 1998 \\
\hline Total receipts & 112.3 & 107.2 & 89.2 & 113.7 & 117.8 & 147.0 \\
\hline Operating receipts & 102.4 & 69.2 & 73.4 & 96.9 & 105.6 & 133.4 \\
\hline Sales of goods and services & 97.9 & 64.2 & 69.9 & 92.0 & 101.8 & 129.9 \\
\hline Current subsidies & 4.5 & 4.9 & 3.5 & 4.9 & 3.8 & 3.6 \\
\hline Other & 9.9 & 38.0 & 15.8 & 16.8 & 12.2 & 13.6 \\
\hline Current expenditures & 96.4 & 74.8 & 68.1 & 93.3 & 99.9 & 141.0 \\
\hline Operating expenditures & 73.0 & 37.2 & 40.1 & 57.9 & 70.3 & 108.6 \\
\hline Of which: Wages and salaries & 4.8 & 5.5 & 6.8 & 8.1 & 9.5 & 8.2 \\
\hline Other & 23.3 & 37.7 & 28.0 & 35.4 & 29.6 & 32.4 \\
\hline Of which: Interest payments & 11.1 & 12.1 & 14.2 & 12.2 & 12.9 & 19.1 \\
\hline $\begin{array}{l}\text { Tax payments to the nationa } \\
\text { government } \\
\text { Interest on national }\end{array}$ & 8.6 & 11.1 & 6.4 & 4.5 & 3.0 & 3.2 \\
\hline government advances & 0.5 & 0.6 & 0.0 & 0.9 & 1.5 & 0.7 \\
\hline Dividend payments & 0.6 & 11.1 & 0.5 & 10.5 & 4.7 & 1.3 \\
\hline Capital expenditures & 41.6 & 42.0 & 22.3 & 31.6 & 35.8 & 44.0 \\
\hline Acquisition of fixed capital assets & 33.9 & 39.5 & 23.8 & 26.9 & 30.5 & 31.3 \\
\hline Change in inventories & 1.1 & 1.5 & 0.5 & 2.8 & 3.2 & 10.5 \\
\hline Other & 6.6 & 1.0 & -2.0 & 2.0 & 2.1 & 2.2 \\
\hline Internal cash generation & 16.0 & 32.3 & 21.1 & 20.4 & 17.8 & 6.0 \\
\hline Financing requirement & 25.6 & 9.7 & 1.3 & 11.2 & 17.9 & 38.0 \\
\hline External financing (net) & 21.9 & 15.4 & 4.5 & 16.1 & 12.3 & 16.8 \\
\hline Domestic financing (net) & 3.8 & -5.7 & -3.2 & -4.9 & 5.7 & 21.2 \\
\hline National government equity $1 /$ & 5.6 & 1.5 & 0.5 & 1.2 & 0.1 & 0.6 \\
\hline National government net lending $1 /$ & 1.5 & 1.9 & 0.8 & -0.4 & 0.3 & 0.5 \\
\hline Domestic bank credits (net) & 1.4 & 0.8 & -3.7 & -3.4 & -1.1 & 12.5 \\
\hline Other domestic financing (net) & -4.8 & -9.9 & -0.9 & -2.3 & 6.3 & 7.6 \\
\hline
\end{tabular}

Source: Data provided by the Philippine authorities.

1/ Taken from corporations' accounts; may differ from national government accounts. 
Table 18. Philippines: Cash Flows of Fourteen Major Public Corporations, 1993-98

(In billions of pesos)

\begin{tabular}{|c|c|c|c|c|c|c|}
\hline & 1993 & 1994 & 1995 & 1996 & 1997 & 1998 \\
\hline Internal cash generation & $\mathbf{1 6 . 0}$ & 32.3 & 21.1 & 20.4 & 17.8 & 6.0 \\
\hline National Power Corporation & 7.7 & 14.5 & 12.3 & 15.3 & 10.7 & 4.9 \\
\hline Philippine National Oil Corporation & 0.9 & 13.9 & 4.7 & -6.6 & -2.2 & -1.5 \\
\hline Metropolitan Water and Sewerage System & 1.2 & 1.2 & 0.6 & 0.9 & 1.4 & 0.8 \\
\hline National Irrigation Administration & 0.0 & -0.1 & -0.1 & 0.0 & 0.1 & 0.1 \\
\hline National Development Company & 1.8 & -0.1 & 0.3 & 3.3 & 3.1 & 0.2 \\
\hline Light Rail Transit Authority & 0.2 & 0.3 & 0.2 & 0.2 & 0.6 & 0.5 \\
\hline National Water Utilities Administration & 0.4 & 0.5 & 0.5 & 0.6 & 0.2 & 0.2 \\
\hline National Electrification Authority & 0.4 & 0.1 & 0.0 & 0.2 & -0.2 & -0.1 \\
\hline National Housing Authority & 0.8 & 0.7 & 1.1 & 2.3 & 0.7 & 0.6 \\
\hline Philippine National Railways & 0.0 & 0.0 & 0.1 & 0.1 & 0.2 & -0.2 \\
\hline Philippine Ports Authority & 1.0 & 1.3 & 1.1 & 1.7 & 1.0 & 1.9 \\
\hline National Food Authority & 1.6 & 0.1 & 0.2 & 2.7 & 2.0 & -1.4 \\
\hline Philippine Economic Zone Authority & 0.0 & 0.0 & 0.2 & 0.0 & 0.2 & 0.2 \\
\hline Metro Manila Transit Corporation I/ & -0.1 & 0.0 & $\ldots$ & $\ldots$ & $\ldots$ & $\ldots$ \\
\hline Capital expenditure & 41.6 & 42.0 & 22.3 & 31.6 & 35.8 & 44.0 \\
\hline National Power Corporation & 28.4 & 35.0 & 16.8 & 19.2 & 24.9 & 21.0 \\
\hline Philippine National Oil Corporation & 5.9 & 4.1 & 4.9 & 4.3 & 1.4 & 1.5 \\
\hline Metropolitan Water and Sewerage System & 1.2 & 0.9 & 0.8 & 1.2 & 1.0 & 1.2 \\
\hline National Irrigation Administration & 0.0 & 0.0 & 0.0 & 0.0 & 0.0 & 0.0 \\
\hline National Development Company & 4.7 & 0.0 & -3.1 & 0.0 & 0.5 & 1.0 \\
\hline Light Rail Transit Authority & 0.1 & 0.2 & 0.3 & 0.5 & 0.6 & 4.2 \\
\hline National Water Utilities Administration & 0.3 & 0.4 & 0.5 & 0.7 & 0.7 & 0.6 \\
\hline National Electrification Authority & 0.7 & 0.1 & 0.0 & 0.8 & 1.1 & 1.0 \\
\hline National Housing Authority & 0.6 & 0.5 & 0.2 & 0.3 & 0.6 & 1.4 \\
\hline Philippine National Railways & 0.2 & 0.3 & 0.6 & 0.6 & 0.6 & 0.2 \\
\hline Philippine Ports Authority & 1.0 & 1.0 & 1.0 & 0.7 & 1.0 & 1.3 \\
\hline National Food Authority & -1.9 & -0.5 & 0.1 & 3.1 & 3.4 & 10.5 \\
\hline Philippine Economic Zone Authority & 0.4 & 0.3 & 0.3 & 0.2 & 0.0 & 0.0 \\
\hline Metro Manila Transit Corporation 1/ & -0.1 & 0.0 & $\ldots$ & $\cdots$ & $\cdots$ & $\cdots$ \\
\hline Balance (Financing Requirement) & -25.6 & -9.7 & -1.3 & -11.2 & -17.9 & -38.0 \\
\hline National Power Corporation & -20.7 & -20.5 & -4.5 & -3.9 & -14.2 & -16.2 \\
\hline Philippine National Oil Corporation & -5.0 & 9.8 & -0.2 & -10.9 & -3.6 & -3.0 \\
\hline Metropolitan Water and Sewerage System & 0.0 & 0.2 & -0.2 & -0.4 & 0.4 & -0.4 \\
\hline National Irrigation Administration & 0.0 & -0.1 & -0.1 & 0.0 & 0.1 & 0.1 \\
\hline National Development Company & -2.9 & -0.1 & 3.4 & 3.2 & 2.6 & -0.8 \\
\hline Light Rail Transit Authority & 0.1 & 0.1 & -0.1 & -0.2 & 0.0 & -3.8 \\
\hline National Water Utilities Administration & 0.0 & 0.2 & 0.0 & -0.1 & -0.5 & -0.4 \\
\hline National Electrification Authority & -0.3 & 0.0 & 0.0 & -0.7 & -1.3 & -1.1 \\
\hline National Housing Authority & 0.2 & 0.2 & 0.9 & 1.9 & 0.1 & -0.8 \\
\hline Philippine National Railways & -0.2 & -0.3 & -0.5 & -0.5 & -0.4 & -0.4 \\
\hline Philippine Ports Authority & 0.0 & 0.3 & 0.1 & 1.0 & 0.0 & 0.6 \\
\hline National Food Authority & 3.6 & 0.6 & 0.1 & -0.5 & -1.4 & -11.9 \\
\hline Philippine Economic Zone Authority & -0.4 & -0.3 & -0.1 & -0.2 & 0.2 & 0.1 \\
\hline Metro Manila Transit Corporation 1/ & 0.0 & 0.0 & $\ldots$ & $\ldots$ & $\ldots$ & $\ldots$ \\
\hline Total financing & 25.6 & 9.7 & 1.3 & 11.2 & 17.9 & 38.0 \\
\hline (In percent of GNP) & 1.7 & 0.6 & 0.1 & 0.5 & 0.7 & 1.4 \\
\hline Net external financing & 21.9 & 15.4 & 4.5 & 16.1 & 12.3 & 16.8 \\
\hline Net domestic financing & 3.8 & -5.7 & -3.2 & .4 .9 & 5.7 & 21.2 \\
\hline Of which: National government transfers & 7.2 & 3.4 & 1.3 & 0.8 & 0.5 & 1.1 \\
\hline
\end{tabular}

Source: Data provided by the Philippine authorities.

1/ Dropped from the list of monitored GOCCs starting September 1994 due to its privatization. 
Table 19. Philippines: Public Sector Operating and Maintenance

Expenditures by Function, 1993-98 1/

(In millions of pesos)

\begin{tabular}{|c|c|c|c|c|c|c|}
\hline & 1993 & 1994 & 1995 & 1996 & 1997 & 1998 \\
\hline \multicolumn{7}{|l|}{ National Government } \\
\hline Economic services & 6,990 & 11,730 & 13,835 & 15,857 & 16,026 & 17,474 \\
\hline Agriculture and agrarian & 2,312 & 3,457 & 4,905 & 6,603 & 6,691 & 4,221 \\
\hline National resources & 700 & 1,612 & 1,441 & 1,178 & 1,631 & 1,318 \\
\hline Trade and industry & 353 & 900 & 885 & 962 & 1,022 & 1,055 \\
\hline Tourism & 70 & 165 & 149 & 111 & 129 & 147 \\
\hline Power and energy & 266 & 389 & 235 & 213 & 187 & 333 \\
\hline Water, transportation and & & & & & & \\
\hline communications & 2,968 & 4,407 & 5,512 & 5,251 & 4,954 & 6,906 \\
\hline Others & 321 & 800 & 708 & 1,539 & 1,412 & 3,494 \\
\hline Social services & 8,257 & 12,079 & 12,125 & 12,027 & 15,280 & 21,974 \\
\hline $\begin{array}{l}\text { Education, culture, and manpower } \\
\text { development }\end{array}$ & 4,783 & 6,709 & 6,961 & 7,062 & 8,185 & 11,328 \\
\hline Health & 2,846 & 3,696 & 3,081 & 3,216 & 4,443 & 5,306 \\
\hline Social security, labor and housing & 526 & 1,504 & 1,910 & 1,482 & 2,262 & 2,088 \\
\hline Others & 102 & 170 & 173 & 266 & 389 & 3,251 \\
\hline Other services $2 /$ & 13,748 & 23,027 & 20,991 & 20,818 & 20,544 & 24,911 \\
\hline Total National Government & 28,995 & 46,836 & 46,951 & 48,702 & 51,850 & 64,359 \\
\hline \multicolumn{7}{|l|}{ Economic and } \\
\hline social services & 15,255 & 23,810 & 25,959 & 27,884 & 31,306 & 39,448 \\
\hline (In percent of GNP) & 1.0 & 1.2 & 1.3 & 1.2 & 1.2 & 1.4 \\
\hline \multicolumn{7}{|l|}{ Major public corporations } \\
\hline \multicolumn{7}{|l|}{ Metropolitan Water and Sewerage } \\
\hline System & 520 & 644 & 727 & 1,612 & 1,202 & 1,019 \\
\hline Philippine National Oil Corporation & 82 & $4,2643 /$ & 3,174 & 1,691 & 2,810 & 3,6354 \\
\hline National Irrigation Administration & 137 & 200 & 218 & 200 & 231 & 242 \\
\hline National Food Authority & 406 & 732 & 504 & 914 & 1,169 & 2,895 \\
\hline
\end{tabular}

Source: Data provided by the Philippine authorities.

1/ Derived by applying the functional classification of obligation expenditures to cash disbursements.

2/ Defense and general public services.

3/ Includes expenses related to the privatization of Petron Corporation.

4/ Includes repayment of accounts payable from 1996 and 1997. 
Table 20. Philippines: Functional Classification of National Government Expenditures, 1993-1998 1/

\begin{tabular}{|c|c|c|c|c|c|c|}
\hline & 1993 & 1994 & 1995 & 1996 & 1997 & 1998 \\
\hline & \multicolumn{6}{|c|}{ (In billions of pesos) } \\
\hline General public services & 40.4 & 56.1 & 60.8 & 76.4 & 87.8 & 97.5 \\
\hline Defense 2/ & 20.4 & 21.6 & 25.5 & 29.9 & 27.7 & 28.8 \\
\hline Economic services & 75.9 & 84.8 & 94.7 & 101.6 & 125.0 & 111.6 \\
\hline Agriculture and agrarian reform & 12.6 & 15.4 & 17.1 & 22.6 & 29.7 & 18.7 \\
\hline Natural resources & 3.4 & 4.0 & 4.6 & 5.3 & 9.1 & 6 \\
\hline Trade and industry & 2.5 & 5.0 & 5.9 & 4.2 & 4.7 & 3.2 \\
\hline Power and energy & 6.6 & 5.8 & 3.2 & 1.1 & 1.5 & 0.9 \\
\hline \multicolumn{7}{|l|}{ Water Resource Development } \\
\hline Transportation and communications & 23.2 & 33.5 & 37.9 & $\begin{array}{r}2.0 \\
41.4\end{array}$ & $\begin{array}{r}0.5 \\
45.9\end{array}$ & 44.3 \\
\hline Others & 18.0 & 17.7 & 22.4 & 24.4 & 27.8 & 34.3 \\
\hline Social services & 71.0 & 72.2 & 92.6 & 118.6 & 150.5 & 174.6 \\
\hline \multicolumn{7}{|l|}{ Education, culture, and } \\
\hline Health, nutrition, population control & 7.2 & 7.4 & 7.8 & 10.9 & 13.4 & 13.5 \\
\hline Social security, labor, and employment & 8.4 & 4.6 & 6.4 & 9.7 & 19.2 & 20.7 \\
\hline Housing and community development & 1.4 & 1.2 & 3.1 & 5.0 & 2.3 & 1.6 \\
\hline Other & 14.1 & 16.6 & 18.2 & 20.8 & 25.7 & 34.6 \\
\hline \multirow[t]{2}{*}{ Total } & 207.7 & 234.7 & 273.6 & 326.5 & 390.9 & 412.4 \\
\hline & \multicolumn{6}{|c|}{ (In percent of GNP) } \\
\hline General public services & 2.7 & 3.2 & 3.1 & 3.4 & 3.5 & 3.5 \\
\hline Defense & 1.4 & 1.2 & 1.3 & 1.3 & 1.1 & 1.0 \\
\hline Economic services & 5.1 & 4.9 & 4.8 & 4.5 & 5.0 & 4.0 \\
\hline \multirow[t]{2}{*}{ Social services } & 4.7 & 4.2 & 4.7 & 5.2 & 6.0 & 6.2 \\
\hline & \multicolumn{6}{|c|}{ (In percent of total) } \\
\hline General public services & 19.5 & 23.9 & 22.2 & 23.4 & 22.5 & 23.6 \\
\hline Defense & 9.8 & 9.2 & 9.3 & 9.2 & 7.1 & 7.0 \\
\hline Economic services & 36.5 & 36.1 & 34.7 & 31.1 & 32.0 & 27.0 \\
\hline Social services & 34.2 & 30.8 & 33.8 & 36.3 & 38.5 & 42.3 \\
\hline
\end{tabular}

Source: Data provided by the Philippine authorities.

l/ Net of interest payments and net lending.

$2 /$ Derived by applying functional classification of obligation expenditures to cash disbursement. 
Table 21. Philippines: Military Expenditure of the National Government, 1993-98 1/2/

\begin{tabular}{|c|c|c|c|c|c|c|}
\hline & 1993 & 1994 & 1995 & 1996 & 1997 & $19983 /$ \\
\hline & \multicolumn{6}{|c|}{ (In millions of pesos) } \\
\hline Total military expenditure & 21,132 & 24,401 & 30,510 & 32,269 & 37,405 & 38,412 \\
\hline \multicolumn{7}{|l|}{ By economic category } \\
\hline Personnel services & 13,310 & 16,284 & 20,253 & 21,223 & 27,597 & 30,274 \\
\hline Maintenance and other operating expenses & 6,609 & 6,562 & 7,751 & 9,001 & 8,753 & 7,708 \\
\hline Capital outlays & 1,213 & 1,555 & 2,506 & 2,045 & 1,055 & 430 \\
\hline \multicolumn{7}{|l|}{ By functions: } \\
\hline Defense & 20,618 & 23,590 & 29,559 & 30,518 & 28,854 & 28,390 \\
\hline Health & 286 & 492 & 622 & 811 & 559 & 674 \\
\hline Education & 228 & 319 & 329 & 480 & 470 & 428 \\
\hline \multirow[t]{2}{*}{ Others (Social security, housing) } & - & - & - & - & 7,521 & 8,118 \\
\hline & \multicolumn{6}{|c|}{ (In percent of GNP) } \\
\hline Total military expenditure & 1.4 & 1.4 & 1.6 & 1.4 & 1.5 & 1.4 \\
\hline Personnel services & 0.9 & 0.9 & 1.0 & 0.9 & 1.1 & 1.1 \\
\hline Maintenance and other operating expenses & 0.4 & 0.4 & 0.4 & 0.4 & 0.3 & 0.3 \\
\hline \multirow[t]{2}{*}{ Capital outlays } & 0.1 & 0.1 & 0.1 & 0.1 & 0.0 & 0.0 \\
\hline & \multicolumn{6}{|c|}{ (As percentage of total expenditure) } \\
\hline By economic category & 6.74 & 6.61 & 8.20 & 7.75 & 7.61 & 7.27 \\
\hline Personnel services & 4.24 & 4.41 & 5.45 & 5.10 & 5.61 & 5.73 \\
\hline Maintenance and other operating expenses & 2.11 & 1.78 & 2.08 & 2.16 & 1.78 & 1.46 \\
\hline Capital outlays & 0.39 & 0.42 & 0.67 & 0.49 & 0.21 & 0.08 \\
\hline By function & 6.74 & 6.61 & 8.20 & 7.64 & 6.08 & 5.58 \\
\hline Defense & 6.57 & 6.39 & 7.94 & 7.33 & 5.87 & 5.37 \\
\hline Health & 0.09 & 0.13 & 0.17 & 0.19 & 0.11 & 0.13 \\
\hline Education & 0.07 & 0.09 & 0.09 & 0.12 & 0.10 & 0.08 \\
\hline
\end{tabular}

Source: Data provided by the Philippine authorities.

1/ Excludes Philippine National Police which is under the Department of the Interior and local governments as well as the Armed Force Modernization Fund.

2/ Data are in an obligation basis.

3/ Preliminary actual. 
Table 22. Philippines: Summary of Monetary Accounts, 1993-98

(In billions of pesos)

\begin{tabular}{|c|c|c|c|c|c|c|c|c|c|c|c|c|}
\hline & \multirow{2}{*}{$\begin{array}{l}1993 \\
\text { Dec. }\end{array}$} & \multirow{2}{*}{$\begin{array}{l}1994 \\
\text { Dec. }\end{array}$} & \multirow{2}{*}{$\begin{array}{l}1995 \\
\text { Dec. }\end{array}$} & \multirow{2}{*}{$\begin{array}{l}1996 \\
\text { Dec. }\end{array}$} & \multicolumn{4}{|c|}{1997} & \multicolumn{4}{|c|}{1998} \\
\hline & & & & & Mar. & Jun. & Sep. & $\overline{\text { Dec. }}$ & Mar. & Jun. & Sep. & $\overline{\text { Dec. }}$ \\
\hline \multicolumn{13}{|l|}{ Bangko Sentral ng Pilipinas } \\
\hline Reserve money & 171.7 & 182.4 & 212.7 & 243.3 & 223.8 & 211.9 & 218.5 & 266.5 & 223.7 & 205.5 & 187.3 & 239.8 \\
\hline Net foreign assets & 56.7 & 91.8 & 118.4 & 232.3 & 249.8 & 217.6 & 246.8 & 211.9 & 220.1 & 261.2 & 271.8 & 232.7 \\
\hline Net domestic assets & 115.0 & 90.6 & 94.3 & 11.0 & -26.0 & -5.7 & -28.3 & 54.6 & 3.6 & -55.7 & -84.5 & 7.1 \\
\hline Credit to public sector & 182.0 & 151.3 & 156.0 & 135.5 & 62.6 & 104.5 & 83.0 & 151.2 & 137.7 & 151.3 & 127.3 & 138.9 \\
\hline National Government & 166.2 & 139.2 & 151.8 & 133.7 & 60.8 & 101.6 & 80.1 & 148.2 & 134.7 & 148.4 & 124.4 & 134.7 \\
\hline Assistance to financial institutions & 4.2 & 4.0 & 3.2 & 3.2 & 3.2 & 4.7 & 8.9 & 8.2 & 15.6 & 17.8 & 18.0 & 18.2 \\
\hline Total rediscounting & 7.3 & 5.3 & 5.9 & 6.5 & 6.3 & 6.6 & 6.6 & 6.6 & 6.8 & 6.9 & 7.7 & 7.3 \\
\hline Open market instruments $\mathrm{I} /$ & -48.7 & -31.5 & .23 .3 & -91.6 & -61.0 & -82.5 & -30.8 & 12.8 & -20.1 & -51.9 & .80 .3 & -29.2 \\
\hline Other iterns net & -29.8 & -38.5 & -47.5 & -42.7 & .36 .9 & -39.0 & -96.0 & -124.2 & -136.5 & -179.8 & -157.2 & -128.1 \\
\hline \multicolumn{13}{|l|}{ Monetary survey 2} \\
\hline Net foreign assets & 104.7 & 123.6 & 117.9 & 69.9 & 75.1 & -10.7 & -51.6 & -76.9 & -66.1 & -67.1 & 0.6 & 16.5 \\
\hline Net domestic assets & 394.8 & 507.2 & 668.5 & 844.0 & 860.8 & 966.7 & $1,076.0$ & $1,251.3$ & $1,222.4$ & $1,240.9$ & $1,143.4$ & $1,202.8$ \\
\hline Domestic credit & 682.1 & 821.6 & $1,084.0$ & $1,507.7$ & $1,499.7$ & $1,618.1$ & $1,777.4$ & $1,922.9$ & $1,853.6$ & $1,899.2$ & $1,872.3$ & $1,868.9$ \\
\hline Public & 274.1 & 299.8 & 335.3 & 377.2 & 325.9 & 356.4 & 404.0 & 468.2 & 451.1 & 478.5 & 443.0 & 459.7 \\
\hline National Govemment, net & 216.2 & 243.5 & 283.9 & 322.8 & 267.1 & 293.6 & 322.7 & 379.6 & 365.4 & 381.4 & 351.8 & 360.8 \\
\hline Private & 408.0 & 521.7 & 748.7 & $1,130.5$ & $\mathrm{I}, 173.8$ & $1,261.7$ & $1,373.5$ & $1,454.7$ & $1,402.5$ & $1,420.7$ & $1,429.3$ & $1,409.2$ \\
\hline Resident FCDUs 3 ' & -136.2 & -158.8 & -206.7 & -317.6 & -336.1 & -348.8 & .367 .7 & -433.4 & -402.1 & -435.0 & -489.8 & $-477.9^{!}$ \\
\hline Net other items & -151.1 & -155.6 & -208.8 & -346.1 & -302.9 & -302.5 & -333.8 & -238.2 & -229.1 & -223.2 & -239.0 &.-188.1 \\
\hline Total liquidity & 499.5 & 630.8 & 786.4 & 913.9 & 935.8 & 956.1 & $1,024.4$ & $1,174.4$ & $1,156.3$ & $1,173.8$ & $1,144.0$ & $1,219.3$ \\
\hline $\mathrm{M} 3$ & 480.3 & 607.6 & 761.4 & 881.4 & 901.8 & 923.4 & 987.7 & $1,066.0$ & $1,057.4$ & $1,082.8$ & $1,065.1$ & $1,144.6$ \\
\hline Narrow money & 133.9 & 152.0 & 184.9 & 222.0 & 216.3 & 224.3 & 222.7 & 258.3 & 241.9 & 251.2 & 238.4 & 281.5 \\
\hline Quasi-money & 341.8 & 451.0 & 570.3 & 652.8 & 678.3 & 692.3 & 755.6 & 795.6 & 803.4 & 820.7 & 818.4 & 856.9 \\
\hline Deposit substitutes & 4.6 & 4.6 & 6.2 & 6.6 & 7.2 & 6.8 & 9.4 & 12.1 & 12.1 & 10.9 & 8.3 & 6.1 \\
\hline Other liabilities & 19.2 & 23.2 & 25.0 & 32.5 & 34.0 & 32.6 & 36.8 & 108.4 & 98.9 & 91.0 & 78.9 & 74.8 \\
\hline
\end{tabular}

Source: Data provided by the Philippine authorities.

1/ Mostly reverse repurchase agreements.

2/ Effective February 1996 Development Bank of the Philippines was reclassified from specialized government bank to commercial bank and hence was included.

3/ Foreign currency deposit units. 
Table 23. Philippines: Factors Affecting Base Money, 1993-98 l/

(In billions of pesos)

\begin{tabular}{|c|c|c|c|c|c|c|c|c|c|c|c|c|}
\hline & \multirow{2}{*}{$\begin{array}{c}1993 \\
\text { Dec }\end{array}$} & \multirow{2}{*}{$\begin{array}{l}1994 \\
\text { Dec. }\end{array}$} & \multirow{2}{*}{$\begin{array}{c}1995 \\
\text { Dec. }\end{array}$} & \multirow{2}{*}{$\begin{array}{r}1996 \\
\text { Dec. }\end{array}$} & \multicolumn{4}{|c|}{1997} & \multicolumn{4}{|c|}{1998} \\
\hline & & & & & Mar. & Jun. & Sep & Dec & Mar. & Jun. & Sep. & Dec. \\
\hline Base money $2 /$ & 175.8 & 196.1 & 229.4 & 262.1 & 243.0 & 238.1 & 276.6 & 304.3 & 290.9 & 273.5 & 259.5 & 316.2 \\
\hline (12-month percentage change) & 14.5 & 11.6 & 17,0 & 14.2 & 9.1 & 8.7 & 28.8 & 16.1 & 19.7 & 14.9 & -6.2 & 3.9 \\
\hline \multicolumn{13}{|l|}{ Stock of reserve eligible securites } \\
\hline outstanding & 4.1 & 41 & 4.2 & 4.3 & 4.2 & 41 & 4.4 & 4.3 & 4.4 & 4.2 & 4.2 & 4.1 \\
\hline Reserve deficiency & 0.0 & 0.0 & 0.0 & 0.0 & 0.0 & 6.3 & 3.4 & 0.0 & 0.0 & 0.0 & 3.9 & 12.5 \\
\hline Government securities & & 9.6 & 125 & 14.5 & 15.0 & 15.8 & 50.3 & 33.5 & 62.8 & 63.8 & 64.1 & 59.8 \\
\hline Reserve money 3 / & 1717 & 182.4 & 212.7 & 243.3 & 223.8 & 2119 & $2 i 85$ & 266.5 & 223.7 & 205.5 & 187.3 & 239.8 \\
\hline Currency issue & 98.0 & 1115 & 130.8 & 150.3 & 132.2 & 130.6 & 135.4 & 181.7 & 153.5 & 153.4 & 144.1 & 385.6 \\
\hline Cash in treasury vault & -0.2 & -0.2 & 0.0 & 0.0 & .0 .1 & 0.0 & 0.0 & 0.0 & 0.0 & 0.0 & 0.0 & 0.0 \\
\hline Reserve balance of banks & 739 & 711 & 81.9 & 92.9 & $91 . ?$ & 81.3 & 83.1 & 84.8 & 70.2 & 52.1 & 43.2 & 54.2 \\
\hline Net foreign assets & 56.7 & 91.8 & 1184 & 232.3 & 249.8 & 217.6 & 246.8 & 211.9 & 220.1 & 261.2 & 271.8 & 232.7 \\
\hline Net international reserves & 99.6 & 129.3 & 1556 & 263.8 & 279.6 & 259.2 & 299.0 & 266.7 & 270.3 & 346.4 & 361.5 & 313.1 \\
\hline Gross intemational reserves & 164.0 & 173.8 & 2037 & 308.8 & 315.2 & 298.4 & 371.4 & 350.5 & 346.8 & 444.7 & 465.4 & 421.6 \\
\hline Short-term foreign liabilities & .64 .4 & -44.5 & -48.1 & -44.9 & -35.6 & -39.2 & -72.4 & .83 .8 & -76.5 & .98 .3 & -103.9 & -108.5 \\
\hline Medium- and long-term foreign liabilities & -42.9 & -37.5 & -37.2 & -31.5 & .29 .8 & -41.6 & -52.2 & .54 .8 & -50.2 & .85 .2 & -89.7 & -80.4 \\
\hline Net domestic assets & 115.0 & 90.6 & 94.3 & 11.0 & -26.0 & -5.7 & -28.3 & 54.6 & 3.6 & .55 .7 & -84.5 & 7.1 \\
\hline Net credit to public sector & 182.0 & 151.3 & 156.0 & 135.5 & 62.6 & 104.5 & 83.0 & 151.2 & 137.7 & 151.3 & 127.3 & 138.9 \\
\hline \multicolumn{13}{|l|}{ Of which: } \\
\hline Net credit to national govermment & .166 .2 & 139.2 & 151.8 & 133.7 & 60.8 & 101.6 & 801 & 148.2 & 134.7 & 148.4 & 124.4 & 134.7 \\
\hline Holding of treasury bills $4 /$ & 171.6 & 120.6 & 137.2 & 139.8 & 74.3 & 1045 & 83.9 & 129.3 & 95.9 & 102.3 & 96.2 & 103.6 \\
\hline Deposits & -113.8 & -84.5 & .73 .4 & .106 .5 & -115.0 & .105 .2 & .96 .9 & .78 .3 & -65.0 & .56 .9 & -63.8 & -59.5 \\
\hline Net credits to 14 corporations & 0.4 & 0.2 & 00 & 0.0 & 0.0 & 0.0 & 0.0 & 0.0 & 0.0 & 0.0 & 0.0 & 0,0 \\
\hline Assistance to financial institutions & 4.2 & 4.0 & 3.2 & 3.2 & 3.2 & 4.7 & 8.9 & 8.2 & 15.6 & 17.8 & 18.0 & 18.2 \\
\hline Total rediscounting & 7.3 & 5.3 & 5.9 & 6.5 & 6.1 & 6.6 & 6.6 & 6.6 & 6.8 & 6.9 & 7.7 & 7.3 \\
\hline Open market instruments $5 /$ & -48.7 & .31 .5 & .23 .3 & -91.6 & -61.0 & -82.5 & -30.8 & 12.8 & .20 .1 & -51.9 & .80 .3 & .29 .2 \\
\hline Other items, net & .29 .8 & -38.5 & .47 .5 & -42.7 & -36.9 & -39.0 & -96.0 & -124.2 & .136 .5 &.-179.8 & -157.2 & -128.1 \\
\hline
\end{tabular}

Source: Data provided by the Philippine authorities.

1/ Foreign assets and liabilities converted into pesos at current exchange rates

2/ Growth rates refer to end period data; therefore they differ from program targets which refer to 10 day test periods

3/ Starting October 4, 1993, deposit balances are exclusive of deposits for Common Trust Funds (CTFs); reserve balances against CTFs are included in other iterns, net

4/ Starting December 1992, excluding Agricultural Loar. Fund investments in treasury bills. 
Table 24. Philippines: Reserve Position of Commercial Banks, 1993-98 1/2/

(In billions of pesos)

\begin{tabular}{|c|c|c|c|c|c|c|c|c|c|c|c|c|}
\hline & \multirow{2}{*}{$\begin{array}{l}1993 \\
\text { Dec. }\end{array}$} & \multirow{2}{*}{$\begin{array}{l}1994 \\
\text { Dec }\end{array}$} & \multirow{2}{*}{$\begin{array}{l}1995 \\
\text { Dec. }\end{array}$} & \multirow{2}{*}{$\begin{array}{l}1996 \\
\text { Dec. }\end{array}$} & \multicolumn{4}{|c|}{1997} & \multicolumn{3}{|c|}{1998} & \multirow[b]{2}{*}{ Dec. } \\
\hline & & & & & Mar. & Jun. & Sep. & Dec. & Mar. & Jun. & Sep. & \\
\hline Required reserves & 89.7 & 99.3 & 111.8 & 130.0 & 126.7 & 130.0 & 165.6 & 157.0 & 158 & 142 & 141 & 168.8 \\
\hline Available reserves 4/ & 91.3 & 101.1 & 118.4 & 138.6 & 135.5 & 123.7 & 162.2 & 159.2 & 163.3 & 146 & 137.1 & 156.3 \\
\hline Cash on hand & 14.2 & 15.5 & 19.7 & 26.9 & 24.6 & 22.6 & 24.4 & 36.5 & 25.9 & 25.9 & 25.6 & 38.2 \\
\hline \multicolumn{13}{|l|}{ Reserves balances with the } \\
\hline Central Bank 5/ & 72.8 & 71.9 & 82.0 & 92.9 & 91.7 & 81.2 & 83.1 & 84.9 & 70.2 & 52.1 & 43.2 & 54.2 \\
\hline Reserve-eligible securities & 4.1 & 4.3 & 4.2 & 4.3 & 4.2 & 4.1 & 4.4 & 4.3 & 4.4 & 4.2 & 4.2 & 4.1 \\
\hline Government securities & $\ldots$ & 9.6 & 12.5 & 14.5 & 15.0 & 15.8 & 50.3 & 33.5 & 62.8 & 63.8 & 64.1 & 59.8 \\
\hline Excess (deficiency) & 1.4 & 1.8 & 6.6 & 8.6 & 8.8 & -6.3 & -3.4 & 2.2 & 5.3 & 4 & -3.9 & -12.5 \\
\hline
\end{tabular}

Sources: Data provided by the Philippine authorities; and staff estimates.

Against deposits and deposit substitutes.

2/ Includes the Land Bank of the Philippines.

3/ Effective September 12,1997, commercial banks' liquidity reserve requirement was reduced to 6 percent and to 4 percent by November 15, 1997 (the liquidity reserve requirement had been increased from 2 percent in July to 8 percent in August).

4. Excludes cash and reserve-eligible securities in excess of 75 percent of required reserves.

5/ Includes centrai bank-supported securities, Central Bank Certificates of Indebtedness, Premyo Savings Bonds, foreign exchange due from banks, and government securities purchased under resale agreements. 
Table 25. Philippines: Public Sector Securities Outstanding, 1993-98

(In billions of pesos)

\begin{tabular}{|c|c|c|c|c|c|c|c|c|c|c|c|c|}
\hline & \multirow{2}{*}{$\begin{array}{l}1993 \\
\text { Dec. }\end{array}$} & \multirow{2}{*}{$\begin{array}{l}1994 \\
\text { Dec. }\end{array}$} & \multirow{2}{*}{$\begin{array}{l}1995 \\
\text { Dec. }\end{array}$} & \multirow{2}{*}{$\begin{array}{l}1996 \\
\text { Dec. }\end{array}$} & \multicolumn{4}{|c|}{1997} & \multicolumn{4}{|c|}{1998} \\
\hline & & & & & Mar. & Jun. & Sep. & Dec. & Mar. & Jun. & Sep. & Dec. \\
\hline National government securities & 624.2 & 605.9 & 662.1 & 706.4 & 704.2 & 697.9 & 694.8 & 708.7 & 711.2 & 747.5 & 745.8 & 789.2 \\
\hline Treasury bills & 413.3 & 368.2 & 391.2 & 393.3 & 386.3 & 384.5 & 386.4 & 392.2 & 403.7 & 430.1 & 422.4 & 443.1 \\
\hline Auctioned 1/ & 203.9 & 172.8 & 158.6 & 166.3 & 161.3 & 161.4 & 143.1 & 133.2 & 133.2 & 137.2 & 120.6 & 113.6 \\
\hline Negotiated $2 /$ & 209.4 & 195.4 & 232.6 & 227.0 & 225.0 & 223.1 & 243.3 & 259.0 & 270.6 & 292.9 & 301.8 & 329.6 \\
\hline Treasury notes & 148.5 & 175.5 & 183.8 & 226.3 & 231.2 & 230.8 & 225.8 & 234.1 & 224.9 & 234.9 & 240.9 & 258.1 \\
\hline Treasury bonds & 61.1 & 61.1 & 85.9 & 85.9 & 85.9 & 82.3 & 82.3 & 82.3 & 82.3 & 82.3 & 82.3 & 82.3 \\
\hline Other & 1.3 & 1.1 & 1.2 & 0.9 & 0.9 & 0.3 & 0.3 & 0.1 & 0.2 & 0.2 & 0.2 & 5.6 \\
\hline Public corporations & 4.1 & 4.5 & 5.2 & 4.8 & 5.1 & 5.1 & 5.1 & 5.8 & 6.1 & 6.3 & 6.6 & 6.8 \\
\hline Monetary institutions & 24.8 & 4.6 & 0.6 & 0.3 & 0.2 & 0.1 & 0.0 & 0.0 & 0.0 & 0.0 & 0.0 & 0.0 \\
\hline Total securities outstanding & 653.1 & 615.0 & 668.0 & 711.4 & 709.5 & 703.1 & 699.9 & 714.5 & 717.3 & 753.8 & 752.4 & 796.1 \\
\hline
\end{tabular}

Sources: Data provided by the Philippine authorities; and staff estimates.

$1 /$ Including tap series.

2 Starting end-December 1993, includes treasury bills issued to the central bank as part of restructuring (special series for BSP and GOCCs.)

3/ Mainly converted from blocked peso accounts. 
Table 26. Philippines: Monetary Survey, 1993-98 1/

\begin{tabular}{|c|c|c|c|c|c|c|c|c|c|c|c|c|}
\hline & \multirow{2}{*}{$\begin{array}{l}1993 \\
\text { Dec. }\end{array}$} & \multirow{2}{*}{$\begin{array}{l}1994 \\
\text { Dec. }\end{array}$} & \multirow{2}{*}{$\begin{array}{l}1995 \\
\text { Dec. }\end{array}$} & \multirow{2}{*}{$\begin{array}{l}1996 \\
\text { Dec. }\end{array}$} & \multicolumn{4}{|c|}{1997} & \multicolumn{4}{|c|}{1998} \\
\hline & & & & & Mar. & Jun. & Sep. & Dec. & Mar. & Jun. & Sep. & Dec. \\
\hline Total liquidity & 499.5 & 630.8 & 786.4 & 913.9 & 935.8 & 956.1 & 1024.4 & 1174.4 & 1156.3 & 1173.8 & 1144.0 & 1219.3 \\
\hline M3 & 480.3 & 607.6 & 761.4 & 881.4 & 901.8 & 923.4 & 987.7 & 1066.0 & 1057.4 & 1082.8 & 1065]$. & 1144.6 \\
\hline Narrow money & 133.9 & 152.0 & 184.9 & 222.0 & 216.3 & 224.3 & 222.7 & 258.3 & 241.9 & 251.2 & 238.4 & 281.5 \\
\hline Quasi-money & 341.8 & 451.0 & 570.3 & 652.8 & 678.3 & 692.3 & 755.6 & 795.6 & 803.4 & 820.7 & 818.4 & 856.9 \\
\hline Deposit substitutes & 4.6 & 4.6 & 6.2 & 6.6 & 7.2 & 6.8 & 9.4 & 12.1 & 12.1 & 10.9 & 8.3 & 6.1 \\
\hline Other liabilities & 19.2 & 23.2 & 25.0 & 32.5 & 34.0 & 32.6 & 36.8 & 108.4 & 98.9 & 91.0 & 78.9 & 74.8 \\
\hline Net foreign assets & 104.7 & 123.6 & 117.9 & 69.9 & 75.1 & -10.7 & -51.6 & -76.9 & -66.1 & -67.1 & 0.6 & 16.5 \\
\hline Net domestic assets & 394.8 & 507.2 & 668.5 & 844.0 & 860.8 & 966.7 & 1076.0 & 1251.3 & 1222.4 & 1240.9 & 1143.4 & 1202.8 \\
\hline Domestic credit & 682.1 & 821.6 & 1084.0 & 1507.7 & 1499.7 & 1618.1 & 1777.4 & 1922.9 & 1853.6 & 1899.2 & 1872.3 & 1868.9 \\
\hline Public & 274.1 & 299.8 & 335.3 & 377.2 & 325.9 & 356.4 & 404.0 & 468.2 & 451.1 & 478.5 & 443.0 & 459.7 \\
\hline National government & 216.2 & 243.5 & 283.9 & 322.8 & 267.1 & 293.6 & 322.7 & 379.6 & 365.4 & 381.4 & 351.8 & 360.8 \\
\hline Other & 57.8 & 56.3 & 51.4 & 54.4 & 58.8 & 62.8 & 81.2 & 88.6 & 85.7 & 97.1 & 91.2 & 98.9 \\
\hline Private & 408.0 & 521.7 & 748.7 & 1130.5 & 1173.8 & 1261.7 & 1373.5 & 1454.7 & 1402.5 & 1420.7 & 1429.3 & 3409.2 \\
\hline Resident FCDUs $2 f$ & -136.2 & -158.8 & -206.7 & -317.6 & -336.1 & .348 .8 & .367 .7 & -433.4 & -402.1 & -435.0 & -489.8 & -477.9 \\
\hline Net other items & -151.1 & -155.6 & -208.8 & -346.1 & -302.9 & -302.5 & -333.8 & -238.2 & -229.1 & -223.2 & -239.0 & -188.1 \\
\hline M3 & 24.6 & 26.5 & 25.3 & 15.8 & 17.3 & 18.9 & 23.5 & 20.9 & 17.3 & 17.3 & 7.8 & 7.4 \\
\hline Net domestic assets & 22.6 & 27.2 & 31.7 & 26.3 & 20.8 & 30.7 & 40.6 & 48.2 & 42.0 & 28.4 & 6.3 & -3.9 \\
\hline Private sector credit & 37.6 & 28.2 & 43.5 & 51.0 & 36.7 & 33.1 & 33.8 & 28.7 & 19.5 & 12.6 & 4.1 & -3.1 \\
\hline $\mathrm{M} 3$ & 31.6 & 35.0 & 38.9 & 38.6 & 35.3 & 36.2 & 38.7 & 39.1 & 41.7 & 37.5 & 36.9 & 37.5 \\
\hline Currency in circulation & 5.5 & 5.5 & 5.7 & 5.4 & 4.2 & 4.2 & 4.3 & 4.5 & 5.6 & 4.6 & 4.5 & 4.5 \\
\hline Net foreign assets & 6.6 & 7.1 & 6.0 & 3.1 & 2.9 & .0 .4 & -2.0 & -1.9 & -3.0 & -2.7 & -3.4 & -2.3 \\
\hline Net domestic assets & 26.2 & 29.2 & 34.1 & 37.0 & 33.7 & 37.9 & 42.1 & 42.4 & 49.0 & 43.6 & 43.4 & 43.3 \\
\hline Private sector credit & 26.8 & 30.0 & 38.2 & 49.5 & 46.0 & 49.4 & 53.8 & 54.4 & 57.0 & 52.1 & 50.5 & 49.7 \\
\hline
\end{tabular}

Source: Data provided by the Philippine authorities.

1/ Effective February 1996 Development Bank of the Philippines was reclassified from specialized government bank to commercial bank and hence was included in the monetary survey.

2/ Foreign currency deposit units. 
Table 27. Philippines: Selected Interest Rates, 1993-98 1/

(In percent)

\begin{tabular}{|c|c|c|c|c|c|c|c|c|c|c|c|c|}
\hline & \multirow{2}{*}{$\begin{array}{l}1993 \\
\text { Dec. }\end{array}$} & \multirow{2}{*}{$\begin{array}{r}1994 \\
\text { Dec. }\end{array}$} & \multirow{2}{*}{$\begin{array}{l}1995 \\
\text { Dec. }\end{array}$} & \multirow{2}{*}{$\begin{array}{r}1996 \\
\text { Dec. }\end{array}$} & \multicolumn{4}{|c|}{1997} & \multicolumn{3}{|c|}{1998} & \multirow[b]{2}{*}{ Dec } \\
\hline & & & & & Mar. & Jun. & Sep. & $\overline{\text { Dec. }}$ & Mar. & Jun. & Sep. & \\
\hline \multicolumn{13}{|c|}{ (End of period) } \\
\hline \multicolumn{13}{|l|}{ Manila reference rates } \\
\hline MRR 60 & 14.8 & 9.8 & 11.4 & 11.8 & 11.0 & 9.9 & 13.6 & 17.3 & 16.8 & 13.9 & 13.9 & 13.7 \\
\hline MRR 90 & 15.0 & 8.8 & 10.3 & 11.1 & 10.3 & 9.6 & 11.5 & 14.1 & 14.7 & 12 & 12.2 & 13.4 \\
\hline Average of all maturities & 14.7 & 9.6 & 11.1 & 11.7 & 10.8 & 9.8 & 13.4 & 17.1 & 16.8 & 13.9 & 13.8 & 13.6 \\
\hline Average lending rate & 16.4 & 13.4 & 14.6 & 14.5 & 13.8 & 13.2 & 17.4 & 20.7 & 20.9 & 17.8 & 17.7 & 18.3 \\
\hline 91-day treasury bill rate & 15.9 & 10.3 & 12.0 & 11.6 & 10.5 & 10.5 & 13.9 & 16.7 & 15.8 & 15 & 13.8 & 13.5 \\
\hline Reverse RP (term) & 0.0 & 14.0 & 13.4 & 11.1 & 10.5 & 10.7 & 13.8 & 12.5 & 13 & 14.6 & 14.2 & 13.6 \\
\hline Interbank call loan rate & 24.1 & 12.0 & 14.1 & 11.2 & 10.5 & 13.2 & 19.6 & 21.4 & 13.2 & 13.3 & 15.5 & 14 \\
\hline \multicolumn{13}{|c|}{ (Period average) } \\
\hline \multicolumn{13}{|l|}{ Manila reference rates } \\
\hline MRR 60 & 9.9 & 8.6 & 9.9 & 11.8 & 10.6 & 10.3 & 15.1 & 18.1 & 17.6 & 14.1 & 14.1 & 13.7 \\
\hline MRR 90 & 13.2 & 8.9 & 8.8 & 11.0 & 9.4 & 9.9 & 12.4 & 14.8 & 15.3 & 12.7 & 12.6 & 13,3 \\
\hline MRR 180 & 12.2 & 8.4 & 9.4 & 10.3 & 8.4 & 8.7 & 11.6 & 13.4 & 13.7 & 11.8 & 12 & 13 \\
\hline Average of all maturities & 12.9 & 8.6 & 9.8 & 11.6 & 10.4 & 10.2 & 14.9 & 17.9 & 17.4 & 14.1 & 14.1 & 13.6 \\
\hline Average lending rate & 14.8 & 13.4 & 13.7 & 14.8 & 13.7 & 13.3 & 18.2 & 20.4 & 20.1 & 16 & 14.6 & 14.8 \\
\hline 91-day treasury bill rate & 12.5 & 12.7 & 11.7 & 11.7 & 9.5 & 11.0 & 15.3 & 17.7 & 16.597 & 14.004 & 13.822 & 13.43 \\
\hline Reverse $R P$ (term) rate & 0.0 & 10.4 & 12.3 & 11.1 & 10.2 & 10.5 & 13.9 & 12 & 16.1 & 14.5 & 13.9 & 13.5 \\
\hline Interbank call loan rate & 19.2 & 10.4 & 12.1 & 11.2 & 10.2 & 14.4 & 15.7 & 13.6 & 13.3 & 13.3 & 15.5 & 13.9 \\
\hline
\end{tabular}

Source: Data provided by the Philippine authorities.

1/ End-period data refer to the average for the last week of each period. 
Table 28. Philippines: Balance of Payments, 1997-2004 1/

(In millions of U.S. dollars)

\begin{tabular}{|c|c|c|c|c|c|c|c|c|c|}
\hline & 1997 & 1998 & $\begin{array}{r}1999 \\
\text { Prog. } \\
\text { EBS/99/6 }\end{array}$ & $\begin{array}{r}1999 \\
\text { Prog. } \\
\text { Revised }\end{array}$ & $\begin{array}{l}2000 \\
\text { Proj. }\end{array}$ & $\begin{array}{l}2001 \\
\text { Proj. }\end{array}$ & $\begin{array}{l}2002 \\
\text { Proj. }\end{array}$ & $\begin{array}{l}2003 \\
\text { Proj. }\end{array}$ & $\begin{array}{l}2004 \\
\text { Proj }\end{array}$ \\
\hline Trade balance & $-11,127$ & .28 & $-2,322$ & 684 & -194 & $-1,270$ & $-2,463$ & -3.802 & $-5,296$ \\
\hline (in percent of GNP) & -13.0 & 0.0 & -3.0 & 0.9 & -0.2 & -1.4 & -2.5 & -3.6 & -4.6 \\
\hline Exporls, f.o.b. & 25,228 & 29,496 & 34,100 & 33,919 & 39,023 & 44,810 & 51,451 & 59,008 & 67,563 \\
\hline Imports, f.o.b. & 36,355 & 29,524 & 36,422 & 33,235 & 39,217 & 46,080 & 53,914 & 62.810 & 72,859 \\
\hline Imports, non-oil & 33,314 & 27,614 & 33,960 & 30,773 & 36,125 & 42,258 & 49,147 & 56,835 & $6 \$, 370$ \\
\hline Imports, oil & 3,041 & 1,910 & 2,462 & 2,462 & 3,092 & $3 ; 822$ & 4,767 & 5,975 & 7,489 \\
\hline Services (net) & 5,696 & 887 & 1,908 & 591 & 457 & 773 & 1,281 & 1,689 & 2,116 \\
\hline Service receipts & 22,835 & 13,881 & 15,812 & 14,726 & 15,651 & 16,642 & 17,703 & 18,868 & 20,139 \\
\hline Remitiances/FCD withdrawals & 11,748 & 8,328 & 9,311 & 8,911 & 9,267 & 9,638 & 10,023 & 10,425 & 10,842 \\
\hline Other & 11,087 & 5,553 & 6.501 & 5,815 & 6,384 & 7,004 & 7,680 & 8,443 & 9,297 \\
\hline Service payments & 17,139 & 12,994 & 13,904 & 14,135 & 15,194 & 15,869 & 16,422 & 17,179 & 18,023 \\
\hline Interest & 2,567 & 2,516 & 2,924 & 2,986 & 3,299 & 3,184 & 2,859 & 2,589 & 2,296 \\
\hline Other & 14,572 & 10,478 & 10,980 & 11,149 & 11,895 & 12,685 & 13,563 & 14,590 & 15,727 \\
\hline Transfers (net) & 1,080 & 435 & 858 & 389 & 369 & 356 & 345 & 333 & 323 \\
\hline Current account & $-4,351$ & 1,294 & 444 & 1,664 & 632 & -141 & -837 & $-1,780$ & $-2,857$ \\
\hline (In percent of GNP) & -5.1 & 1.9 & 0.6 & 2.1 & 0.7 & -0.2 & 0.9 & -1.7 & -2.5 \\
\hline Financial account & 988 & 65 & 1,168 & 1,496 & 1,795 & 2,496 & 3,928 & 5,852 & 6,977 \\
\hline Foreign investment & 762 & 1,672 & 1,493 & 1,713 & 1,499 & 1,626 & 1,761 & 1,904 & 2,194 \\
\hline Direct investment & 1,113 & 1,592 & 1,193 & 938 & 695 & 786 & 879 & 979 & 1,080 \\
\hline Portolio investment & -351 & 80 & 300 & 775 & 804 & 840 & 882 & 925 & 1,114 \\
\hline MLT loans and bonds & 4,148 & 1,768 & 2,498 & 3,311 & 1,516 & 787 & 1,267 & 1,433 & 1,786 \\
\hline Inflows & 7,724 & 5,791 & 6,765 & 7,617 & 6,411 & 7,241 & $7,6] 1$ & 7,627 & 8,068 \\
\hline Outflows $1 /$ & 3,576 & 4,023 & 4,267 & 4,306 & 4,895 & 6,454 & 6,344 & 6,194 & 6,282 \\
\hline Short-term, including trade-related & 495 & $-1,521$ & $-1,270$ & $-1,003$ & 738 & 1,284 & 1,042 & 1,109 & 1,146 \\
\hline Commercial banks' NFA (increase -) $2 \prime$ & 1,188 & $-1,320$ & -836 & $-1,522$ & $-2,108$ & $-1,317$ & -262 & 1,282 & 1,722 \\
\hline Other (1ncluding errors and omissions) $3 /$ & $-5,605$ & -534 & -717 & $-1,003$ & 150 & 116 & 120 & 124 & 129 \\
\hline $\begin{array}{l}\alpha / w: \text { Commercial banks' NFA operations } \\
\text { on account of BSP (increase -) }\end{array}$ & 0 & 370 & -825 & $-1,120$ & 0 & 0 & 0 & 0 & 1 \\
\hline Overall balance & $.3,363$ & 1,359 & 1,612 & 3,160 & 2,427 & 2,355 & 3,091 & 4,072 & 4,120 \\
\hline Net international reserves (increase -) & 3,363 & $-1,359$ & $-1,612$ & $-3,160$ & $-2,427$ & $-2,355$ & $-3,091$ & $-4,072$ & $-4,120$ \\
\hline BSP gross reserves (increase -) & 2,977 & $-2,038$ & $-2,309$ & $-3,927$ & $-2,679$ & $-2,346$ & $-2,600$ & $-3,199$ & $-3,552$ \\
\hline Fund credit (nel) & 435 & 651 & 697 & 683 & 252 & -9 & -491 & -873 & -568 \\
\hline Purchases & 700 & 730 & 752 & 737 & 261 & 0 & 0 & 0 & 0 \\
\hline Repurchases & -265 & .79 & -55 & .54 & -8 & -9 & .491 & .873 & -568 \\
\hline Change in other BSP liabiluties & -49 & 28 & 0 & 84 & 0 & 0 & 0 & 0 & 0 \\
\hline \multicolumn{10}{|l|}{ Memorandum items: } \\
\hline BSP gross reserves & 8,768 & 10,806 & 12,900 & 14,733 & 17,412 & 19,758 & 22,358 & 25,557 & 29,109 \\
\hline (In months of imports of goods and services) & 2.0 & 3.0 & 3.1 & 3.7 & 3.8 & 3.8 & 3.8 & 3.8 & 3.8 \\
\hline BSP adjusted gross reserves $4 /$ & 7,568 & 9,606 & 11,700 & 13,408 & 16,087 & 18,433 & 21,033 & 24,232 & 27,784 \\
\hline (In months of imports of goods and services) & 1.7 & 2.7 & 2.8 & 3.4 & 3.5 & 3.6 & 3.6 & 3.6 & 3.7 \\
\hline BSP NIR & 6,672 & 8,031 & 9,444 & 11,191 & 13,618 & 15,972 & 19,063 & 23,135 & 27,255 \\
\hline Monitored extemal debt $5 /$ & 50,728 & 53,509 & 57,804 & 56,419 & 58,493 & 60,959 & 64,270 & 69,218 & 76,911 \\
\hline (In percent of GNP) & 59.3 & 75.5 & 74.6 & 71.0 & 68.8 & 67.3 & 65.8 & 65.6 & 67.5 \\
\hline Short-tem debt & 11,514 & 10,245 & 11,600 & 9,703 & $\ldots$ & $\ldots$ & $\ldots$ & $\ldots$ & ... \\
\hline (In percent of adjusted reserves) & 152.1 & 106.7 & 99.1 & 72.4 & $\ldots$ & $\ldots$ & $\ldots$ & $\ldots$ & .. \\
\hline Debt-service ratio $6 /$ & 119 & 12.8 & 12.3 & 12.8 & 14.1 & 14.9 & 13.4 & 11.9 & 10.1 \\
\hline Export volume (percent change) & 23.1 & 19.5 & 11.5 & 12.7 & 13.8 & 13.7 & 13.6 & 13.5 & 13.4 \\
\hline Import volume (percent change) & 16.4 & -12.8 & 7.7 & 10.5 & 15.9 & 15.2 & 14.4 & 13.7 & 13.4 \\
\hline Export value (percent change) & 22.8 & 16.9 & 15.6 & $\mathbf{1 5 . 0}$ & 15.0 & 14.8 & 14.8 & 14.7 & 14.5 \\
\hline Import value (percent change) & 14.0 & -18.8 & 14.6 & 12.6 & 18.0 & 17.5 & 17.0 & 16.5 & 16.0 \\
\hline
\end{tabular}

Sources: Philippine authorities and staff estimates.

1/ Includes net repurchase of Philippine bonds in secondary markets by residents.

2/ Commercial banks' NFA differ from the monetary survey due to differences in coverage. Excludes FCDU and NDF operations.

3/ Includes FCDU and NDF operations, and errors and omissions. The $\$ 750$ ING repayment is also included.

4) Gross reserves less gold and securities pledged as collateral against short-term liabilities.

5/ Monitored extemal liabilities are defined as external debt plus ljabilities of foreign banks in the Philippines to their headquarters, branches, and agencies, some extemal debt not registered with the central bank, and private capital lease arrangements.

$6 /$ In percent of exports of goods and services. 
Table 29. Philippines: External Indicators, 1993 -98

\begin{tabular}{|c|c|c|c|c|c|c|}
\hline & 1993 & 1994 & 1995 & 1996 & 1997 & 1998 \\
\hline & \multicolumn{4}{|c|}{ (In millions of U.S. dollars, end period) } & & \\
\hline $\begin{array}{l}\text { Gross official reserves l/ } \\
\text { (In months of imports }\end{array}$ & 4,721 & 6,372 & 6,662 & 10,445 & 7,568 & 9,606 \\
\hline of goods and services) & 2.5 & 2.7 & 2.2 & 2.8 & 1.7 & 2.7 \\
\hline \multicolumn{7}{|l|}{ Net international reserves } \\
\hline of the central bank & 3,496 & 5,297 & 5,928 & 10,035 & 6,671 & 8,031 \\
\hline \multicolumn{7}{|c|}{ (In percent of GNP) } \\
\hline Exports & 20.6 & 20.5 & 22.9 & 23.8 & 29.4 & 43.2 \\
\hline Imports & 31.8 & 32.5 & 34.6 & 37.0 & 42.4 & 43.3 \\
\hline Trade balance & -11.2 & -11.9 & -11.7 & -13.1 & -13.0 & 0.0 \\
\hline Current account & -5.5 & -4.5 & -4.3 & -4.6 & -5.1 & 1.9 \\
\hline \multicolumn{7}{|c|}{ (Annual percent change) } \\
\hline Export volume & 13.2 & 14.0 & 17.0 & 20.7 & 23.1 & 19.5 \\
\hline Export unit price & 2.3 & 4.0 & 10.6 & -2.4 & -7.0 & -2.0 \\
\hline Import volume & 19.9 & 18.6 & 14.5 & 22.8 & 16.4 & -12.8 \\
\hline Import unit price & 1.1 & 2.2 & 8.0 & -1.6 & -6.2 & -7.2 \\
\hline Terms of trade & 1.2 & 1.8 & 2.4 & -0.8 & -0.9 & 5.5 \\
\hline \multicolumn{7}{|l|}{ Memorandum items: } \\
\hline \multicolumn{7}{|l|}{ Peso/dollar exchange rate } \\
\hline Annual average & 27.1 & 26.4 & 25.7 & 26.2 & 29.5 & 40.9 \\
\hline End-of-period & 27.7 & 24.4 & 26.2 & 26.3 & 40.0 & 39.1 \\
\hline
\end{tabular}

Source: Data provided by the Philippine authorities and staff estimates.

1/ Adjusted for gold- and security-backed borrowing. 
Table 30. Philippines: Volume, Unit Prices, and Values of Principal Exports, 1993-98

(Unless otherwise indicated, volumes in thousands of metric tons,

unit prices in U.S. dollars per ton, and values in millions of U.S. dollars)

\begin{tabular}{|c|c|c|c|c|c|c|}
\hline & 1993 & 1994 & 1995 & 1996 & 1997 & $\begin{array}{r}1998 \\
\text { Prelim. }\end{array}$ \\
\hline \multicolumn{7}{|l|}{ Coconut oil } \\
\hline Volume & 859 & 849 & 1,339 & 793 & 1,109 & 1,178 \\
\hline Price & 416 & 560 & 616 & 720 & 607 & 599 \\
\hline Value & 358 & 475 & 826 & 571 & 673 & 706 \\
\hline \multicolumn{7}{|l|}{ Desiccated coconut } \\
\hline Volume & 93 & 75 & 73 & 70 & 77 & 72 \\
\hline Price & 897 & 934 & 933 & 1,220 & 1,152 & 1,016 \\
\hline Value & 84 & 70 & 68 & 85 & 88 & 73 \\
\hline \multicolumn{7}{|l|}{ Copra meal/cake } \\
\hline Volume & 488 & 574 & 756 & 475 & 570 & 540 \\
\hline Price & 93 & 92 & 88 & 119 & 92 & 65 \\
\hline Value & 45 & 53 & 67 & 56 & 53 & 35 \\
\hline \multicolumn{7}{|l|}{ Sugar } \\
\hline Volume & 324 & 182 & 153 & 318 & 198 & 185 \\
\hline Price & 314 & 333 & 430 & 429 & 418 & 432 \\
\hline Value & 102 & 61 & 66 & 136 & 83 & 80 \\
\hline \multicolumn{7}{|l|}{ Lumber } \\
\hline Volume $(000 \mathrm{cu} . \mathrm{m})$ & 89 & 41 & 87 & 146 & 144 & 44 \\
\hline Price (US\$ $/ \mathrm{cu} . \mathrm{m})$ & 292 & 236 & 209 & 171 & 178 & 227 \\
\hline Value & 26 & 10 & 18 & 25 & 26 & 10 \\
\hline \multicolumn{7}{|l|}{ Plywood } \\
\hline Volume $(000 \mathrm{cu} . \mathrm{m})$ & 40 & 10 & 17 & 12 & 14 & 6 \\
\hline Price (US $\$ / c u . m$ ) & 435 & 428 & 341 & 362 & 363 & 333 \\
\hline Value & 17 & 4 & 6 & 5 & 5 & 2 \\
\hline \multirow{2}{*}{\multicolumn{7}{|c|}{ Copper concentrates }} \\
\hline & & & & & & 101 \\
\hline Price & 327 & 385 & 476 & 329 & 371 & 248 \\
\hline Value & 109 & 113 & 134 & 52 & 44 & 25 \\
\hline \multicolumn{7}{|l|}{ Copper metal } \\
\hline Volume & 137 & 137 & 120 & 126 & 96 & 102 \\
\hline Price & 1,933 & 1,963 & 2,843 & 2,351 & 2,405 & 1,737 \\
\hline Value & 264 & 270 & 341 & 297 & 232 & 178 \\
\hline \multicolumn{7}{|l|}{ Gold } \\
\hline Volume (troy oz.) & 151 & 176 & 416 & 202 & 149 & 114 \\
\hline Price (US\$/troy oz.) & 339 & 370 & 370 & 270 & 330 & 298 \\
\hline Value & 51 & 65 & 62 & 55 & 49 & 34 \\
\hline \multicolumn{7}{|l|}{ Iron ore } \\
\hline Volume & 3,919 & 4,666 & 4,692 & 4,435 & 4,718 & 3,381 \\
\hline Price & 16 & 16 & 16 & 16 & 19 & 18 \\
\hline Value & 62 & 74 & 74 & 70 & 90 & 60 \\
\hline \multicolumn{7}{|l|}{ Bananas } \\
\hline Volume & 1,153 & 1,155 & 1,213 & 1,253 & 1,150 & 1,140 \\
\hline Price & 196 & 186 & 184 & 188 & 188 & 190 \\
\hline Value & 226 & 215 & 224 & 237 & 217 & 216 \\
\hline Fish, fresh or preserved (value) & 343 & 379 & 378 & 295 & 291 & 306 \\
\hline Other nonmanufactured (value) & 756 & 824 & 934 & 1,065 & 1,114 & 861 \\
\hline Garments (value) & 2,272 & 2,375 & 2,570 & 2,423 & 2,349 & 2,356 \\
\hline Electronics (value) & 3,551 & 4,984 & 7,413 & 9,988 & 13,052 & 17,156 \\
\hline Other manufactured (value) & 2,906 & 3,256 & 3,885 & 4,684 & 6,087 & 6,354 \\
\hline \multirow{2}{*}{$\begin{array}{l}\text { Special transactions } \\
\text { and re-exports (value) }\end{array}$} & & & & & & \\
\hline & 203 & 255 & 381 & 499 & 775 & 1,044 \\
\hline Total exports & 11,375 & 13,483 & 17,447 & 20,543 & 25,228 & 29,496 \\
\hline
\end{tabular}

Source: Data provided by the Philippine authorities. 
Table 31. Philippines: Composition of Imports, 1993-98

\begin{tabular}{|c|c|c|c|c|c|c|}
\hline & 1993 & 1994 & 1995 & 1996 & 1997 & $\begin{array}{l}1998 \\
\text { Est. }\end{array}$ \\
\hline & \multicolumn{6}{|c|}{ (In millions of U.S. dollars) } \\
\hline Mineral fuels and lubricants & 2,016 & 2,040 & 2,461 & 3,008 & 3,074 & 2,021 \\
\hline Oil & 1,316 & 1,308 & 1,931 & 2,458 & 2,458 & 1,433 \\
\hline Volume (mn. bbls.) & 83 & 86 & 117 & 130 & 127 & 114 \\
\hline Price $(\$ / \mathrm{bbl}$.) & 16 & 15 & 17 & 19 & 19 & 13 \\
\hline Nonfuel imports & 15,581 & 19,293 & 23,930 & 28,877 & 33,281 & 27,503 \\
\hline Capital goods & 5,610 & 6,868 & 8,029 & 10,472 & 14,369 & 12,051 \\
\hline Raw materials and intermediate goods & 7,855 & 9,606 & 12,174 & 14,058 & 14,634 & 11,586 \\
\hline Consumer goods & 1,587 & 2,109 & 2,784 & 3,331 & 3,091 & 2,621 \\
\hline Special transactions $1 /$ & 529 & 710 & 943 & 1,016 & 1,187 & 1,245 \\
\hline \multirow[t]{2}{*}{ Total } & 17,597 & 21,333 & 26,391 & 31,885 & 36,355 & 29,524 \\
\hline & \multicolumn{6}{|c|}{ (In percent of total) } \\
\hline Mineral fuels and lubricants & 11.5 & 9.6 & 9.3 & 9.4 & 8.5 & 6.8 \\
\hline Nonfuel imports & 88.5 & 90.4 & 90.7 & 90.6 & 91.5 & 93.2 \\
\hline Capital goods & 31.9 & 32.2 & 30.4 & 32.8 & 39.5 & 40.8 \\
\hline Raw materials and intermediate goods & 44.6 & 45.0 & 46.1 & 44.1 & 40.3 & 39.2 \\
\hline Consumer goods & 9.0 & 9.9 & 10.5 & 10.4 & 8.5 & 8.9 \\
\hline Special transactions $1 /$ & 3.0 & 3.3 & 3.6 & 3.2 & 3.3 & 4.2 \\
\hline Total & 100.0 & 100.0 & 100.0 & 100.0 & 100.0 & 100.0 \\
\hline
\end{tabular}

Source: Data provided by the Philippine authorities.

1/ Imports on consignment (for export use) 
Table 32. Philippines: Nonmerchandise Trade, 1993-98

(In millions of U.S. dollars)

\begin{tabular}{|c|c|c|c|c|c|c|}
\hline Item & 1993 & 1994 & 1995 & 1996 & 1997 & $\begin{array}{c}1998 \\
\text { Prelim. }\end{array}$ \\
\hline Nonmerchandise trade, net & 2,507 & 3,964 & 4,765 & 6,800 & 5,696 & 887 \\
\hline Earnings & 7,497 & 10,550 & 14,374 & 19,006 & 22,835 & 13,881 \\
\hline Freight and merchandise insurance & 156 & 185 & 252 & 331 & 391 & 294 \\
\hline Other transportation & 71 & 54 & 29 & 34 & 36 & 38 \\
\hline Travel & 1,178 & 973 & 1,124 & 1,546 & 2,341 & 1,418 \\
\hline Investment income & 548 & 773 & 1,147 & 1,753 & 1,956 & 1,499 \\
\hline Profits, earnings, and dividends & 70 & 116 & 344 & 700 & 589 & 302 \\
\hline Interest income & 478 & 657 & 803 & 1,053 & 1,367 & 1,197 \\
\hline Central bank & 219 & 322 & 345 & 476 & 568 & 587 \\
\hline Commercial banking system & 259 & 335 & 458 & 577 & 799 & 610 \\
\hline Government & 56 & 19 & 24 & 18 & 7 & 12 \\
\hline Nonmerchandise insurance & 12 & 4 & 54 & 21 & 16 & 16 \\
\hline Personal income & 2,276 & 3,009 & 3,869 & 4,306 & 5,742 & 4,932 \\
\hline Peso conversions of FCDs & 1,680 & 2,815 & 4,827 & 5,863 & 6,006 & 3,410 \\
\hline Commissions and fees & 173 & 207 & 164 & 373 & 260 & 181 \\
\hline Construction activity & 10 & 6 & 10 & 18 & 27 & 37 \\
\hline Operating expenses & 476 & 695 & 673 & 822 & 1,214 & 539 \\
\hline Other services & 861 & 1,810 & 2,201 & 3,921 & 4,901 & 1,505 \\
\hline Payments & 4,990 & 6,586 & 9,609 & 12,206 & 17,139 & 12,994 \\
\hline Freight and merchandise insurance & 1,176 & 1,300 & 1,950 & 2,285 & 2,602 & 1,886 \\
\hline Other transportation & 278 & 181 & 132 & 40 & 113 & 109 \\
\hline Travel & 130 & 196 & 422 & 1,266 & 1,935 & 1,950 \\
\hline Investment expense & 1,887 & 1,919 & 2,671 & 2,720 & 3,017 & 2,919 \\
\hline Profits, earnings, and dividends & 326 & 311 & 469 & 470 & 394 & 318 \\
\hline Reinvested earnings & 43 & 29 & 23 & 44 & 56 & 85 \\
\hline Interest expenses & 1,518 & 1,579 & 2,179 & 2,206 & 2,567 & 2,516 \\
\hline Government & 18 & 15 & 20 & 37 & 49 & 20 \\
\hline Nonmerchandise insurance & 64 & 77 & 76 & 42 & 68 & 31 \\
\hline Personal income & 13 & 13 & 15 & 57 & 0 & 0 \\
\hline Commissions and fees & 150 & 275 & 340 & 775 & 623 & 551 \\
\hline Construction activity & 23 & 23 & 65 & 1,186 & 511 & 218 \\
\hline Other services & 1,251 & 2,587 & 3,918 & 3,798 & 8,221 & 5,310 \\
\hline
\end{tabular}

Source: Data provided by the Philippine authorities. 
Table 33. Philippines: Direction of Trade, 1993-98

(In percent)

\begin{tabular}{|c|c|c|c|c|c|c|c|c|c|c|c|c|}
\hline & \multicolumn{6}{|c|}{ Exports, f.o.b. } & \multicolumn{6}{|c|}{ Imports, f.o.b. } \\
\hline & 1993 & 1994 & 1995 & 1996 & 1997 & $\begin{array}{r}1998 \\
\text { Prelim. }\end{array}$ & 1993 & 1994 & 1995 & 1996 & 1997 & $\begin{array}{r}1998 \\
\text { Prelim. }\end{array}$ \\
\hline United States & 38.4 & 38.1 & 35.3 & 33.9 & 34.9 & 34.2 & 20.0 & 18.5 & 18.4 & 18.5 & 19.7 & 21.8 \\
\hline Japan & 16.0 & 15.0 & 15.7 & 17.9 & 16.6 & 14.3 & 22.9 & 24.3 & 22.6 & 22.4 & 20.4 & 20.4 \\
\hline $\begin{array}{l}\text { European Union, of which: } \\
\text { Germany } \\
\text { Netherlands } \\
\text { United Kingdom } \\
\text { France }\end{array}$ & $\begin{array}{r}18.2 \\
5.2 \\
3.2 \\
4.8 \\
1.8\end{array}$ & $\begin{array}{r}18.0 \\
4.9 \\
3.8 \\
4.7 \\
1.4\end{array}$ & $\begin{array}{r}18.0 \\
4.0 \\
4.6 \\
5.3 \\
1.2\end{array}$ & $\begin{array}{r}17.9 \\
4.1 \\
5.4 \\
4.6 \\
1.1\end{array}$ & $\begin{array}{r}18.5 \\
4.2 \\
6.6 \\
4.3 \\
0.9\end{array}$ & $\begin{array}{r}20.9 \\
3.5 \\
7.9 \\
6.0 \\
0.9\end{array}$ & $\begin{array}{r}12.8 \\
3.5 \\
1.4 \\
2.2 \\
1.1\end{array}$ & $\begin{array}{r}10.3 \\
3.6 \\
1.3 \\
1.8 \\
1.1\end{array}$ & $\begin{array}{r}10.2 \\
3.5 \\
1.2 \\
2.1 \\
1.2\end{array}$ & $\begin{array}{r}14.0 \\
3.8 \\
1.0 \\
1.5 \\
1.0\end{array}$ & $\begin{array}{r}14.9 \\
3.2 \\
1.4 \\
1.5 \\
3.4\end{array}$ & $\begin{array}{r}11.0 \\
2.9 \\
0.8 \\
1.1 \\
1.7\end{array}$ \\
\hline $\begin{array}{l}\text { Middle East, of which: } \\
\text { Saudi Arabia } \\
\text { Kuwait } \\
\text { Iraq }\end{array}$ & $\begin{array}{l}2.3 \\
0.6 \\
0.1 \\
0.0\end{array}$ & $\begin{array}{l}1.8 \\
0.4 \\
0.1 \\
0.0\end{array}$ & $\begin{array}{l}1.3 \\
0.4 \\
0.1 \\
0.0\end{array}$ & $\begin{array}{l}1.2 \\
0.3 \\
0.1 \\
0.0\end{array}$ & $\begin{array}{l}0.9 \\
0.1 \\
0.1 \\
0.0\end{array}$ & $\begin{array}{l}0.8 \\
0.1 \\
0.0 \\
0.0\end{array}$ & $\begin{array}{l}8.4 \\
4.2 \\
1.1 \\
0.0\end{array}$ & $\begin{array}{l}7.4 \\
4.4 \\
0.4 \\
0.0\end{array}$ & $\begin{array}{l}8.5 \\
6.2 \\
0.1 \\
0.0\end{array}$ & $\begin{array}{l}8.5 \\
5.2 \\
0.1 \\
0.0\end{array}$ & $\begin{array}{l}7.3 \\
2.9 \\
0.0 \\
0.1\end{array}$ & $\begin{array}{l}5.9 \\
1.9 \\
0.1 \\
0.1\end{array}$ \\
\hline $\begin{array}{l}\text { ASEAN, of which: } 1 / \\
\text { Indonesia } \\
\text { Malaysia } \\
\text { Singapore }\end{array}$ & $\begin{array}{l}3.7 \\
0.4 \\
1.4 \\
3.3\end{array}$ & $\begin{array}{l}5.4 \\
0.5 \\
1.6 \\
5.3\end{array}$ & $\begin{array}{l}7.9 \\
0.7 \\
1.8 \\
5.7\end{array}$ & $\begin{array}{l}8.5 \\
0.7 \\
3.3 \\
6.0\end{array}$ & $\begin{array}{l}7.2 \\
0.8 \\
2.5 \\
6.4\end{array}$ & $\begin{array}{l}6.7 \\
0.4 \\
3.9 \\
6.2\end{array}$ & $\begin{array}{l}5.3 \\
1.9 \\
2.0 \\
5.6\end{array}$ & $\begin{array}{l}4.9 \\
1.7 \\
2.0 \\
6.8\end{array}$ & $\begin{array}{l}6.0 \\
2.2 \\
2.2 \\
5.9\end{array}$ & $\begin{array}{l}7.0 \\
2.0 \\
2.5 \\
5.5\end{array}$ & $\begin{array}{l}7.4 \\
1.9 \\
2.6 \\
6.0\end{array}$ & $\begin{array}{l}9.2 \\
2.0 \\
3.2 \\
5.9\end{array}$ \\
\hline Australia & 1.0 & 1.0 & 0.8 & 0.8 & 0.8 & 0.6 & 2.7 & 2.8 & 2.8 & 2.5 & 2.6 & 2.3 \\
\hline Hong Kong & 4.8 & 4.8 & 4.7 & 4.2 & 4.6 & 4.5 & 5.0 & 5.2 & 4.8 & 4.3 & 4.3 & 4.4 \\
\hline Korea & 1.9 & 2.2 & 2.5 & 1.8 & 1.9 & 1.7 & 5.1 & 5.2 & 5.2 & $5: 2$ & 6.0 & 7.4 \\
\hline BRO 2/ & 0.1 & 0.1 & 0.1 & 0.1 & 0.0 & 0.0 & 0.5 & 0.9 & 1.3 & 1.5 & 1.1 & 0.6 \\
\hline China & 1.5 & 1.2 & 1.2 & 1.6 & 1.0 & 1.1 & 1.0 & 1.4 & 2.2 & 2.1 & 2.4 & 4.1 \\
\hline Other & 12.0 & 12.3 & 12.5 & 12.1 & 13.5 & 15.1 & 16.3 & 19.3 & 18.0 & 14.0 & 13.8 & 12.9 \\
\hline All countries & 100.0 & 100.0 & 100.0 & 100.0 & 100.0 & 100.0 & 100.0 & 100.0 & 100.0 & 100.0 & 100.0 & 100.0 \\
\hline
\end{tabular}

Source: Data provided by the Philippine authorities.

1/ Association of South-East Asian Nations.

2/ Baltics, Russia and other. 
Table 34. Philippines: International Reserves of the Banking System, 1993-98

(In millions of U.S. dollars; end of period)

\begin{tabular}{lrrrrrr}
\hline & 1993 & 1994 & 1995 & 1996 & 1997 & 1998 \\
& & & & & & \\
\hline & & & & & & \\
& & & & & & \\
Monetary authorities (net) & 3,496 & 5,297 & 5,928 & 10,035 & 6,672 & 8,031 \\
$\quad$ Assets & 5,922 & 7,122 & 7,762 & 11,745 & 8,768 & 10,806 \\
$\quad$ Liabilities & 2,426 & 1,825 & 1,834 & 1,710 & 2,096 & 2,776 \\
$\quad$ of which: IMF & 1,219 & 1,064 & 728 & 405 & 889 & 1,562 \\
& & & & & & \\
Commercial banks (net) 1/ & 912 & 238 & $-1,336$ & $-5,838$ & $-7,415$ & $-6,392$ \\
$\quad$ Assets & 4,008 & 5,028 & 5,170 & 6,803 & 6,440 & 6,432 \\
$\quad$ Liabilities & 3,096 & 4,790 & 6,506 & 12,641 & 13,855 & 12,824 \\
& & & & & & \\
Banking system & 4,408 & 5,535 & 4,592 & 4,197 & -743 & 1,638 \\
$\quad$ Assets & 9,930 & 12,150 & 12,932 & 18,548 & 15,208 & 17,238 \\
$\quad$ Liabilities & 5,522 & 6,615 & 8,340 & 14,351 & 15,951 & 15,600 \\
& & & & & & \\
\hline
\end{tabular}

Source: Data provided by the Philippine authorities.

1/ Monetary claims on, and liabilities to, nonresidents. 
Table 35. Philippines: Total External Debt, 1993-98

$\begin{array}{llllll}1993 & 1994 & 1995 & 1996 & 1997 & 1998\end{array}$

\author{
External debt \\ Short term \\ Of which: commercial banks \\ Medium and long term \\ Of which: commercial banks \\ IMF \\ Total \\ Monitored external liabilities 1/ \\ Short term \\ Of which: commercial banks \\ Medium and long term \\ Of which: commercial banks \\ IMF \\ Total
}

External debt

Short term

Of which: commercial banks

Medium and long term

Of which: commercial banks

IMF

Monitored external liabilities 1/

Short term

Of which: commercial banks

Medium and long term

Of which: commercial banks

IMF

External debt

Short term

Of which: commercial banks

Medium and long term

Of which: commercial banks

IMF

Total

Monitored external liabilities $1 /$

Short term

Of which: commercial banks

Medium and long term

Of which: commercial banks

IMF

Total (in millions of U.S. dollars)

$\begin{array}{rrrrrr}5,035 & 5,197 & 5,279 & 7,207 & 8,439 & 7,185 \\ 383 & 1,156 & 1,800 & 3,245 & 3,611 & 3,571 \\ 29,188 & 32,387 & 33,274 & 34,668 & 36,122 & 39,070 \\ 1,627 & 2,133 & 2,440 & 2,789 & 3,770 & 4,208 \\ 1,312 & 1,139 & 814 & 405 & 872 & 1,562 \\ 35,535 & 38,723 & 39,367 & 42,280 & 45,433 & 47,817 \\ & & & & & \\ \ldots & & & & & \\ \ldots & \ldots & \ldots & \ldots & 11,513 & 10,245 \\ \ldots & \ldots & \ldots & \ldots & 6,693 & 6,631 \\ \ldots & \ldots & \ldots & \ldots & 38,343 & 41,702 \\ \ldots & \ldots & \ldots & \ldots & 5,066 & 5,436 \\ \ldots & \ldots & \ldots & \ldots & 872 & 1,562 \\ & & \ldots & \ldots & 50,728 & 53,509\end{array}$

(in percent of total external debt)

$\begin{array}{rrrrrr}14.2 & 13.4 & 13.4 & 17.0 & 18.6 & 15.0 \\ 1.1 & 3.0 & 4.6 & 7.7 & 7.9 & 7.5 \\ 82.1 & 83.6 & 84.5 & 82.0 & 79.5 & 81.7 \\ 4.6 & 5.5 & 6.2 & 6.6 & 8.3 & 8.8 \\ 3.7 & 2.9 & 2.1 & 1.0 & 1.9 & 3.3\end{array}$

(in percent of total monitored external liabilities)

$\begin{array}{rrrrrr}\ldots & \ldots & \ldots & \ldots & 22.7 & 19.1 \\ \ldots & \ldots & \ldots & \ldots & 13.2 & 12.4 \\ \ldots & \ldots & \ldots & \ldots & 75.6 & 77.9 \\ \ldots & \ldots & \ldots & \ldots & 10.0 & 10.2 \\ \ldots & \ldots & \ldots & \ldots & 1.7 & 2.9\end{array}$

(in percent of GNP)

$\begin{array}{rrrrrr}9.1 & 7.9 & 6.9 & 8.4 & 9.9 & 10.1 \\ 0.7 & 1.8 & 2.4 & 3.8 & 4.2 & 5.0 \\ 52.8 & 49.3 & 43.7 & 40.2 & 42.2 & 55.1 \\ 2.9 & 3.2 & 3.2 & 3.2 & 4.4 & 5.9 \\ 2.4 & 1.7 & 1.1 & 0.5 & 1.0 & 2.2 \\ 64.2 & 58.9 & 51.7 & 49.0 & 53.1 & 67.5\end{array}$

$\begin{array}{rrrrr}\ldots & \ldots & \ldots & 13.5 & 14.5 \\ \ldots & \ldots & \ldots & 7.8 & 9.4 \\ \ldots & \ldots & \ldots & 44.8 & 58.8 \\ \ldots & \ldots & \ldots & 5.9 & 7.7 \\ \ldots & \ldots & \ldots & 1.0 & 2.2 \\ \ldots & \ldots & \ldots & 59.3 & 75.5\end{array}$

Sources: Data provided by the Philippines authorities; and staff estimates

1/ Monitored external liabilities are defined as external debt plus liabilities of foreign banks in the Philippines to their headquarters, branches, and agencies, some external debt not registered with the central bank, and private capital lease arrangements. 
Table 36. Philippines: External Debt by Type of Debt and Creditor, 1993-98

(in millions of U.S. dollars)

\begin{tabular}{|c|c|c|c|c|c|c|c|c|c|c|c|c|c|c|c|c|c|c|}
\hline & \multicolumn{3}{|c|}{1993} & \multicolumn{3}{|c|}{1994} & \multicolumn{3}{|c|}{1995} & \multicolumn{3}{|c|}{1996} & \multicolumn{3}{|c|}{1997} & \multicolumn{3}{|c|}{ September 1998} \\
\hline & $\begin{array}{l}\text { Medium- } \\
\text { and Long- } \\
\text { Temm }\end{array}$ & $\begin{array}{l}\text { Short } \\
\text { TTerm }\end{array}$ & Tolal & $\begin{array}{l}\text { Medium- } \\
\text { and Long- } \\
\text { Term }\end{array}$ & $\begin{array}{l}\text { Short. } \\
\text { Term }\end{array}$ & Total & $\begin{array}{l}\text { Medium- } \\
\text { and Long- } \\
\text { Term }\end{array}$ & $\begin{array}{l}\text { Short. } \\
\text { Term }\end{array}$ & Total & $\begin{array}{l}\text { Medium- } \\
\text { and Long- } \\
\text { Term }\end{array}$ & $\begin{array}{l}\text { Shoort- } \\
\text { Term }\end{array}$ & Total & $\begin{array}{l}\text { Medium } \\
\text { and Long. } \\
\text { Terr }\end{array}$ & $\begin{array}{l}\text { Short. } \\
\text { Term }\end{array}$ & Total & $\begin{array}{l}\text { Medium } \\
\text { and Long- } \\
\text { Term }\end{array}$ & $\begin{array}{l}\text { Short- } \\
\text { Term }\end{array}$ & Total \\
\hline \multicolumn{19}{|l|}{ Extemal debt } \\
\hline By type of debt & 30,500 & 5,035 & 35.535 & 33.527 & 5,196 & 38.723 & 34.088 & 5.279 & 39.367 & 34,668 & 7,207 & 41,875 & 36,993 & 8.440 & 45,433 & 38,436 & 7,972 & 46,408 \\
\hline Nortmonetary y/ & 28,793 & 3,444 & 32.237 & 31,295 & 3,285 & 34,580 & 31,541 & 2,374 & 33.915 & 30,613 & 2,632 & 33,245 & 31,691 & 3,078 & 34,769 & 33,085 & 1,940 & 35,025 \\
\hline Public 21 & 26,552 & 389 & $26,94]$ & 27,630 & 91 & 27.721 & 26.594 & 70 & 26,664 & 24,110 & 23 & 24,133 & 22,252 & 20 & 22.272 & 22.396 & 41 & 22,437 \\
\hline Privale & 2.241 & 3,055 & 5,296 & 3.665 & 3,194 & 6.859 & 4,947 & 2.304 & 7,251 & 6,503 & 2,609 & 9.112 & 9,439 & 3,058 & 12.497 & 10,689 & 1.899 & 12,588 \\
\hline Barking system & 1,707 & 1.591 & 3,298 & 2.232 & 1,911 & 4.143 & 2,547 & 2,905 & 5.452 & 4,056 & 4,575 & 8.631 & 5,302 & 5,362 & 10.664 & 5,350 & 6,032 & 11,382 \\
\hline Central bark & 80 & 1,208 & 1.288 & 99 & 756 & 855 & 107 & 1,105 & 1,212 & 112 & 1,303 & 3,415 & 795 & 1.704 & 2.499 & 1.475 & 2,204 & 3,679 \\
\hline Commercial banks & 1,627 & 383 & 2,010 & 2.133 & 1,155 & 3.288 & 2.440 & 1.800 & 4,240 & 3.944 & 3,272 & 7,216 & 4,507 & 3.658 & 8.165 & $\begin{array}{l}3.875 \\
\end{array}$ & 3,828 & 7.703 \\
\hline By creditor & 30,500 & 5,035 & 35.535 & 33,525 & 5,197 & 38.722 & 34,089 & 5,279 & 39,368 & 34,668 & 7,207 & 41,875 & 36,994 & 8,439 & 45.433 & 38.436 & 2973 & 46,408 \\
\hline Commercial barks & 2,177 & 2,698 & 4.875 & 2,357 & 2,305 & 4.662 & 1,899 & 3.207 & 5,106 & 2,483 & 4,931 & 7,414 & 3,363 & 5.509 & 8,872 & 4,185 & 4,481 & 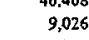 \\
\hline Ober financial instiutrions & 200 & 102 & 302 & 390 & 460 & 850 & 1,122 & 117 & 1,239 & 306 & 653 & 959 & 407 & 897 & 1,304 & 704 & 479 & 1.183 \\
\hline Suppliers' redits & 950 & 2,235 & 3.185 & 1,134 & 2,415 & 3,549 & 946 & 1,642 & 2,588 & 1,065 & 1,523 & 2,588 & 833 & 2,526 & 2.359 & 825 & 1,043 & 1.868 \\
\hline Muthlitateral, of which: & 9,202 & 0 & 9,202 & 9,859 & 0 & 9,859 & 9,617 & 0 & 9,617 & 8,634 & 0 & 8,634 & 8,638 & 0 & 8,638 & 8,980 & 0 & 8,980 \\
\hline World Bark & 4,597 & 0 & 4,597 & 4,855 & 0 & 4,855 & 4.995 & 0 & 4,995 & 4,676 & 0 & 4,676 & 4,146 & 0 & $\begin{array}{r}4,146 \\
406\end{array}$ & $\begin{array}{l}8,056 \\
4,056\end{array}$ & 0 & 4,056 \\
\hline $\mathrm{AsDB}$ & 2,892 & 0 & 2,892 & 3,331 & 0 & 3,331 & 3,331 & 0 & 3,331 & 3,117 & 0 & 3,117 & 3.091 & 0 & 3,091 & 3,069 & 0 & 3,069 \\
\hline $\mathrm{INF}$ & 1,312 & 0 & 1,312 & 1,139 & 0 & 1.139 & 814 & 0 & 814 & 405 & 0 & 405 & 889 & 0 & 889 & 1,162 & 0 & $\begin{array}{l}\text { 1.1602 } \\
1.62\end{array}$ \\
\hline Expor credit agencies. of which: & 3,997 & 0 & 3,997 & 4,487 & 0 & 4.487 & 3,939 & 0 & 3,939 & 4,677 & 0 & 4,677 & 4.718 & 0 & 4,718 & $\begin{array}{l}4,979 \\
4,979\end{array}$ & a & 4,979 \\
\hline Japan & 2,344 & 0 & 2,344 & 3,149 & 0 & 3.149 & 2,645 & 0 & 2,645 & 3,270 & 0 & 3.270 & 3,128 & 0 & 3,128 & 3,102 & 0 & 3.102 \\
\hline United Slates & 154 & 0 & 154 & 755 & 0 & 755 & 695 & 0 & 695 & 588 & 0 & 588 & 715 & 0 & 715 & 996 & 0 & 996 \\
\hline Ober bilateral. of which: & 9,372 & 0 & 9.372 & 10.546 & 0 & 10.546 & 10.454 & 0 & 10,454 & 8.747 & is & 8,762 & 8,519 & 70 & 8.589 & 8.582 & 125 & 8.706 \\
\hline Japan & 7,577 & 0 & 7,577 & 8,694 & 0 & 8.694 & 8,418 & 0 & 8,418 & 6,939 & 0 & 6.939 & 6,262 & 0 & 6.262 & 6.248 & 0 & 6.248 \\
\hline United States & 684 & 0 & 684 & 647 & 0 & 647 & 656 & 0 & 656 & 501 & 0 & 501 & 631 & 0 & 631 & 603 & 0 & 603 \\
\hline Ocher & 4,602 & 0 & 4,602 & 4.752 & 17 & 4.769 & 6.112 & 313 & 6.425 & 8.756 & 85 & 8,841 & 10,516 & 437 & 10,953 & 10.181 & 1.485 & 11,666 \\
\hline \multicolumn{19}{|l|}{ Monitored extemal labbilities $3 /$} \\
\hline Shor term & $\ldots$ & $\ldots$ & $\ldots$ & $\ldots$ & $\ldots$ & .. & $\ldots$ & $\ldots$ & $\ldots$ & $\ldots$ & $\ldots$ & $\ldots$ & $\ldots$ & $\ldots$ & 10,816 & & $\ldots$ & 10,122 \\
\hline of which: cormerciai banks & $\cdots$ & ... & $\cdots$ & $\ldots$ & $\cdots$ & ... & $\cdots$ & $\cdots$ & $\ldots$ & $\ldots$ & $\ldots$ & $\cdots$ & $\cdots$ & $\cdots$ & 6,655 & $\cdots$ & $\ldots$ & 5,732 \\
\hline Medium and long lerm & $\ldots$ & $\ldots$ & $\ldots$ & $\ldots$ & $\ldots$ & .. & $\ldots$ & $\ldots$ & $\ldots$ & $\ldots$ & $\ldots$ & $\ldots$ & $\ldots$ & $\ldots$ & 39,662 & $\ldots$ & ... & 39,383 \\
\hline of which: corrmercial barks & $\cdots$ & $\ldots$ & ... & $\cdots$ & $\ldots$ & $\therefore$ & & $\ldots$ & $\ldots$ & & $\ldots$ & $\ldots$ & $\ldots$ & $\ldots$ & 5,195 & & ... & 4,283 \\
\hline & $\ldots$ & $\ldots$ & $\ldots$ & $\ldots$ & $\ldots$ & $\ldots$ & & & $\ldots$ & $\ldots$ & $\ldots$ & $\ldots$ & $\ldots$ & $\ldots$ & 50.478 & & $\ldots$ & 49.505 \\
\hline Of which: commercial banks & $\cdots$ & $\ldots$ & $\ldots$ & $\ldots$ & $\ldots$ & .. & $\ldots$ & $\ldots$ & $\ldots$ & $\ldots$ & $\ldots$ & $\ldots$ & $\cdots$ & $\ldots$ & 11.850 & $\ldots$ & $\ldots$ & 10,015 \\
\hline \multirow{3}{*}{\multicolumn{19}{|c|}{$\begin{array}{l}\text { Mernorandum items } \\
\text { Gross forcign exchange liabilitites of commercial bantes }\end{array}$}} \\
\hline & & & & & & & & & & & & & & & & & & \\
\hline & & $\ldots$ & & $\ldots$ & ... & & & & & & & & & $\ldots$ & 28.030 & & & 28,720 \\
\hline 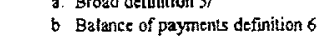 & $\begin{array}{l}\cdots \\
\cdots\end{array}$ & $\begin{array}{l}\cdots \\
\cdots\end{array}$ & $\begin{array}{l}8,000 \\
3,096\end{array}$ & $\ldots$ & $\cdots$ & $\begin{array}{r}4.790 \\
4.790\end{array}$ & & & $\begin{array}{l}14,531 \\
6,506\end{array}$ & $\cdots$ & $\cdots$ & $\begin{array}{l}20,24, \\
12,641\end{array}$ & $\cdots$ & $\cdots$ & $\begin{array}{l}28,000, \\
15,719\end{array}$ & & $\cdots$ & $\begin{array}{l}28,20 \\
16,193\end{array}$ \\
\hline Exemal debr according to the BIS 8 & $\ldots$ & $\ldots$ & $\ldots$ & $\ldots$ & & 6,544 & & $\ldots$ & 8,067 & $\ldots$ & $\ldots$ & 13,351 & $\ldots$ & $\ldots$ & $\ldots$ & $\ldots$ & $\ldots$ & \\
\hline Vis:avvis barks & $\ldots$ & $\ldots$ & $\ldots$ & & & 3.769 & & & 4,951 & $\ldots$ & $\ldots$ & 9,362 & & $\ldots$ & $\ldots$ & $\ldots$ & $\ldots$ & $\ldots$ \\
\hline Vis-2-vis notbanks & $\ldots$ & $\ldots$ & $\cdots$ & & ... & 2.775 & & $\ldots$ & 3,116 & $\ldots$ & $\ldots$ & 3,989 & $\ldots$ & $\ldots$ & $\ldots$ & $\ldots$ & $\ldots$ & \\
\hline
\end{tabular}

Sources: Dala provided by the Philippine authonites, and BIS (intemational Banking and Financial Markel Developments).

1/ Includes prefinancing of exports.

$2 /$ For 1993, includes central bark Bosro of Liquidators

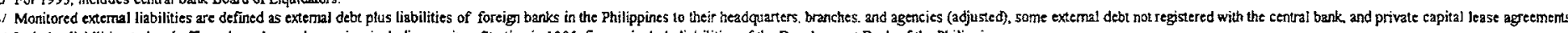
47. Ureludes liabilities to head offices, branches and agencies, including equily. Starting in 1995, figures includec liabilities of che Development Bark of the Philippines

6) Excludes liabilities to residerns

"External position of reporting banks vis-s.vis the Ptilippines. 
Table 37. Philippines: External Debt Service, 1993-98

\begin{tabular}{|c|c|c|c|c|c|c|}
\hline & 1993 & 1994 & 1995 & 1996 & 1997 & 1998 \\
\hline & \multicolumn{6}{|c|}{ (In millions of U.S. dollars) } \\
\hline \multicolumn{7}{|l|}{ Debt service before rescheduling } \\
\hline and accumulation of arrears & 3,594 & 4,907 & 5,032 & 5,026 & 5,597 & 5,061 \\
\hline Principal (excluding IMF) & 2,012 & 3,056 & 2,494 & 2,541 & 2,843 & 2,437 \\
\hline Multilateral & 471 & 566 & 682 & 657 & 633 & 597 \\
\hline Bilateral & 827 & 1,257 & 1,385 & 1,154 & 1,342 & 1,125 \\
\hline Banks and financial institutions $1 /$ & 540 & 843 & 193 & 200 & 590 & 426 \\
\hline Other $2 /$ & 174 & 390 & 234 & 530 & 278 & 289 \\
\hline IMF repurchase & 64 & 272 & 359 & 279 & 186 & 108 \\
\hline Interest & 1,518 & 1,579 & 2,179 & 2,206 & 2,568 & 2,516 \\
\hline Multilateral & 633 & 711 & 634 & 593 & 542 & 505 \\
\hline Bilateral & 642 & 742 & 784 & 634 & 579 & 551 \\
\hline Banks and financial institutions & 152 & 32 & 363 & 550 & 707 & 640 \\
\hline Other & 91 & 94 & 398 & 429 & 740 & 820 \\
\hline Rescheduling & 818 & 478 & 0 & 0 & 0 & 0 \\
\hline Paris Club & 211 & 0 & 0 & 0 & 0 & 0 \\
\hline Commercial banks & 607 & 478 & 0 & 0 & 0 & 0 \\
\hline Change in arrears & 0 & 178 & -178 & 0 & 0 & 0 \\
\hline \multirow[t]{2}{*}{$\begin{array}{l}\text { Debt service after rescheduling } \\
\text { and changes in arrears }\end{array}$} & 3,511 & 4,251 & 4,854 & 5,026 & 5,597 & 5,061 \\
\hline & \multicolumn{6}{|c|}{ (In percent of exports of goods and services) } \\
\hline \multicolumn{7}{|l|}{ Debt-service ratio (including $\mathrm{IMF}$ ) } \\
\hline Before rescheduling & 22.9 & 20.4 & 15.8 & 12.7 & 11.9 & 12.8 \\
\hline $\begin{array}{l}\text { Ratio of interest payments } \\
\text { (before rescheduling) to GNP }\end{array}$ & 2.7 & 2.4 & 2.9 & 2.5 & 3.0 & 3.7 \\
\hline
\end{tabular}

Sources: Data provided by the Philippine authorities; and staff estimates.

1/ Excluding monetary liabilities and debt conversions.

2/ Figures for 1995-97 include some debt-equity and bond-equity conversions, as well as some prepayments. 
Table 38. Philippines: Selected Indicators on the Banking System as of December 31, 1998

(In billions of pesos, unless otherwise indicated)

$\begin{array}{ccccc}\text { Commercial Banks } & & \text { Thrift } & \text { Rural } & \text { Banking } \\ \text { Expanded Non-Exp. Foreign } & \text { Total } & \text { Banks } & \text { Banks 1/ } & \text { System }\end{array}$

Physical composition

Number of banks

Total number of branches

Balance Sheet

Total assets

Of which: Loan portfolio (net)

Deposit liabilities

Of which: Foreign currency

Capital

Annual Income and Expenses

Total operating income Of which: Interest income

Net income before tax

Trust Assets

21
3519

20

650

2028.6
1209.0
1317.9
480.4
258.9

216.8
130.9
140.0
49.7
44.2

$\begin{array}{rr}310.4 & 2555.8 \\ 141.4 & 1481.3 \\ 128.2 & 1586.1 \\ 70.8 & 600.9 \\ 7.8 & 310.9\end{array}$

184.0

166.0

20.6

18.4

25.0

19.5

229.6

203.9

22.4

0.4

3.8

26.6

364.3

22.0

27.4

413.7
117

1357

209.8

124.2

131.7

8.1

28.4

16.7

14.9

0.0

5.5
826

1114

58.2

40.2

37.6

0.0

9.0

2.7

2.1

0.2

249.0

220.9

26.8

419.2

Source: Bangko Sentral ng Pilipinas

1/ Data for rural banks in Sections II and III is for June 30, 1998. 
Table 39. Philippines: Deposit Liabilities of the Banking System, 1993-98

$1993 \quad 1994 \quad 1995 \quad 1996 \frac{1997}{\text { June December }} \frac{1998}{\text { June December }}$

\begin{tabular}{|c|c|c|c|c|c|c|c|c|}
\hline Banking system & 638.9 & 786.1 & 1000.0 & 1270.5 & 1411.0 & 1630.8 & 1708.8 & 1719.7 \\
\hline Commercial banks & 556.8 & 693.1 & 873.8 & 1119.6 & 1232.1 & 1457.9 & 1542.6 & 1550.0 \\
\hline Expanded & & 531.0 & 772.1 & 993.6 & & 1256.0 & 1291.4 & 1317.9 \\
\hline Nonexpanded & & 162.1 & 101.7 & 126.0 & & 201.9 & 251.2 & 232.1 \\
\hline Thrift banks & 51.6 & 65.2 & 91.1 & 120.4 & 143.7 & 136.3 & 129.6 & 132.1 \\
\hline Rural banks $2 /$ & 13.4 & 17.6 & 23.3 & 30.3 & 35.2 & 36.6 & 36.6 & 37.6 \\
\hline Specialized government banks & 17.1 & 10.3 & 11.7 & 0.2 & -- & -. & -- & -. \\
\hline Banking system & 100.0 & 100.0 & 100.0 & 100.0 & 100.0 & 100.0 & 100.0 & 100.0 \\
\hline Commercial banks & 87.2 & 88.2 & 87.4 & 88.1 & 87.3 & 89.4 & 90.3 & 90.1 \\
\hline Expanded & 0.0 & 67.6 & 77.2 & 78.2 & 0.0 & 77.0 & 75.6 & 76.6 \\
\hline Nonexpanded & 0.0 & 20.6 & 10.2 & 9.9 & 0.0 & 12.4 & 14.7 & 13.5 \\
\hline Thrift banks & 8.1 & 8.3 & 9.1 & 9.5 & 10.2 & 8.4 & 7.6 & 7.7 \\
\hline Rural banks $2 /$ & 2.1 & 2.2 & 2.3 & 2.4 & 2.5 & 2.2 & 2.1 & 2.2 \\
\hline Specialized government banks & 2.7 & 1.3 & 1.2 & 0.0 & -- & -- & - & -- \\
\hline Banking system & 29.3 & 23.0 & 27.2 & 27.1 & & 28.4 & 21.1 & 5.5 \\
\hline Commercial banks & 30.5 & 24.5 & 26.1 & 28.1 & & 30.2 & 25.2 & 6.3 \\
\hline Expanded & & & 45.4 & 28.7 & & 26.4 & & 4.9 \\
\hline Nonexpanded & & & -37.2 & 23.9 & & 60.2 & & 15.0 \\
\hline Thrift banks & 24.6 & 26.3 & 39.7 & 32.1 & & 13.2 & -9.8 & -3.1 \\
\hline Rural banks 2/ & 27.7 & 30.8 & 33.0 & 29.7 & & 20.9 & 3.8 & 2.7 \\
\hline Specialized government banks & 10.5 & -39.8 & 13.6 & -98.0 & & - & - & - \\
\hline
\end{tabular}

Sources: Bangko Sentral ng Pilipinas; and Fund staff estimates.

1/ Consolidated with commercial banks after 1996.

2/ Data for December 1998 correspond to June. 
Table 40. Philippines: Loans of the Banking System, 1993 -98

\begin{tabular}{|c|c|c|c|c|c|c|c|c|}
\hline & \multirow[b]{2}{*}{1993} & \multirow[b]{2}{*}{1994} & \multirow[b]{2}{*}{1995} & \multirow[b]{2}{*}{1996} & \multicolumn{2}{|c|}{1997} & \multicolumn{2}{|c|}{1998} \\
\hline & & & & & June & December & June & December \\
\hline & \multicolumn{6}{|c|}{ (Billions of pesos) } & & \\
\hline Total loans & 567.0 & 722.0 & 980.4 & 1377.3 & 1601.7 & 1753.6 & 1772.0 & 1714.2 \\
\hline Commercial banks & 506.4 & 637.2 & 866.3 & 1221.8 & 1419.0 & 1573.2 & 1595.4 & 1542.5 \\
\hline Thrift banks & 45.1 & 65.7 & 89.2 & 122.1 & 145.8 & 139.6 & 135.8 & 130.5 \\
\hline \multirow[t]{2}{*}{ Rural banks $1 /$} & 15.5 & 19.1 & 24.9 & 33.4 & 36.9 & 40.8 & 40.9 & 41.2 \\
\hline & \multicolumn{6}{|c|}{ (12-month growth rates) } & & \\
\hline Total loans & 36.9 & 27.3 & 35.8 & 40.5 & & 27.3 & 10.6 & -2.2 \\
\hline Commercial banks & 38.1 & 25.8 & 36.0 & 41.0 & & 28.8 & 12.4 & -2.0 \\
\hline Thrift banks & 30.1 & 45.8 & 35.7 & 37.0 & & 14.3 & -6.9 & -6.5 \\
\hline Rural ba Rural banks $1 /$ & 22.7 & 23.1 & 30.0 & 34.3 & & 22.2 & 10.7 & 1.0 \\
\hline
\end{tabular}

Sources: Bangko Sentral ng Pilipinas; and Fund staff estimates.

1/ Data for December 1998 correspond to June. 
Table 41. Philippines: Deposits in Commercial Banks' Foreign Currency Deposit Units (FCDUs), 1993-98

\begin{tabular}{|c|c|c|c|c|c|c|c|}
\hline & & & & & \multicolumn{2}{|c|}{1997} & \multirow{2}{*}{$\frac{1998}{\text { June }}$} \\
\hline & 1993 & 1994 & 1995 & $1996^{\circ}$ & June & December & \\
\hline & \multicolumn{7}{|c|}{ (Millions of U.S. dollars) } \\
\hline Residents' deposits & 4914.0 & 6459.7 & 7881.6 & 12079.0 & 13223.8 & 10813.0 & 10348.4 \\
\hline Nonresidents' deposits & 367.9 & 710.9 & 870.9 & 1893.7 & 3120.4 & 3044.2 & 3239.8 \\
\hline \multirow[t]{2}{*}{ Total FCDU deposits } & 5281.9 & 7170.6 & 8752.5 & 13972.8 & 16344.2 & 13857.2 & 13588.3 \\
\hline & \multicolumn{7}{|c|}{ (Growth rates) } \\
\hline Residents' deposits & & 31.5 & 22.0 & 53.3 & 36.1 & -10.5 & -21.7 \\
\hline Nonresidents' deposits & & 93.2 & 22.5 & 117.4 & 144.4 & 60.8 & 3.8 \\
\hline \multirow[t]{2}{*}{ Total FCDU deposits } & & 35.8 & 22.1 & 59.6 & 48.7 & -0.8 & -16.9 \\
\hline & \multicolumn{7}{|c|}{ (Percent of total FCDU deposits) } \\
\hline Residents' deposits & 93.0 & 90.1 & 90.0 & 86.4 & 80.9 & 78.0 & 76.2 \\
\hline Nonresidents' deposits & 7.0 & 9.9 & 10.0 & 13.6 & 19.1 & 22.0 & 23.8 \\
\hline Total FCDU deposits & 100.0 & 100.0 & 100.0 & 100.0 & 100.0 & 100.0 & 100.0 \\
\hline
\end{tabular}

Sources: Bangko Sentral ng Pilipinas; and Fund staff estimates. 
Table 42. Philippines: Indicators of Concentration in the Banking System as of June 30, 1998 (Largest 10 banks in each category as percent of total) $1 /$

\begin{tabular}{|c|c|c|c|c|c|c|c|}
\hline & \multicolumn{4}{|c|}{ Commercial Banks } & \multirow{2}{*}{$\begin{array}{l}\text { Thrift } \\
\text { Banks }\end{array}$} & \multirow{2}{*}{$\begin{array}{l}\text { Rural } \\
\text { Banks }\end{array}$} & \multirow{2}{*}{$\begin{array}{l}\text { Banking } \\
\text { System }\end{array}$} \\
\hline & Expanded & Non-Exp. & Foreign & Total & & & \\
\hline Total resources & 74.4 & 77.6 & 95.0 & 60.8 & 71.5 & 16.7 & 55.2 \\
\hline Total loan portfolio & 72.3 & 76.4 & 94.5 & 61.9 & 73.8 & 16.5 & 55.8 \\
\hline Nonperforming loans (NPLs) & 68.0 & 112.6 & 90.8 & 58.1 & 68.3 & 13.3 & 48.3 \\
\hline NPLs + Restr. loans + ROPOA l/ & 71.1 & 108.6 & 90.5 & 58.9 & 68.7 & 16.9 & 48.7 \\
\hline Loan loss provisions & 77.9 & 69.2 & 95.4 & 68.7 & 84.6 & 11.9 & 60.3 \\
\hline Demand liabilities & 59.0 & 87.2 & 99.5 & 53.8 & 70.8 & 20.3 & 51.6 \\
\hline Capital & 72.7 & 58.0 & 78.2 & 59.3 & 54.9 & 15.2 & 52.5 \\
\hline Net income before tax & 77.6 & -13.0 & 104.2 & 68.0 & -- & -- & 66.8 \\
\hline
\end{tabular}

Source: Bangko Sentral ng Pilipinas; and Fund staff estimates.

1/ Banks ranked according to total resources. 
Table 43. Philippines: Selected Financial Ratios of the Banking System, $1993-98$ (Percentages)

\begin{tabular}{|c|c|c|c|c|c|c|c|}
\hline & \multirow[b]{2}{*}{1993} & \multirow[b]{2}{*}{1994} & \multirow[b]{2}{*}{1995} & \multirow[b]{2}{*}{1996} & \multirow[b]{2}{*}{1997} & \multicolumn{2}{|c|}{1998} \\
\hline & & & & & & June & December \\
\hline \multicolumn{8}{|l|}{ Capital adequacy } \\
\hline Total banking system $1 /$ & 19.2 & 18.6 & 18.8 & 16.8 & 16.0 & 17.1 & 17.6 \\
\hline Commercial banks & 18.6 & 17.9 & 18.7 & 16.6 & 15.9 & 16.9 & 17.5 \\
\hline Specialized govt. banks $2 /$ & 26.2 & 23.5 & 20.4 & -- & -- & -- & -- \\
\hline Thrift banks & 15.0 & 18.2 & 17.9 & 19.4 & 17.6 & 19.6 & 19.8 \\
\hline Rural banks 3/ & 21.9 & 17.9 & 17.4 & 15.8 & 17.4 & 17.4 & 17.6 \\
\hline \multicolumn{8}{|l|}{ Profitability } \\
\hline \multicolumn{8}{|l|}{ Return on assets (ROA) 4/ } \\
\hline Total banking system 1/ & 1.94 & 2.03 & 2.07 & 2.16 & 1.66 & 0.37 & 1.00 \\
\hline Commercial banks & 1.83 & 1.85 & 1.92 & 2.17 & 1.67 & 0.36 & 1.07 \\
\hline Specialized govt. banks 2/ & 3.05 & 4.91 & 4.77 & -. & -- & -- & -- \\
\hline Thrift banks & 2.28 & 2.13 & 2.09 & 1.92 & 1.38 & -0.02 & 0.16 \\
\hline Rural banks & 2.28 & 2.15 & 2.53 & 2.58 & 2.36 & 2.36 & 1.04 \\
\hline \multicolumn{8}{|l|}{ Return on equity (ROE) $5 /$} \\
\hline Total banking system $/ /$ & 15.1 & 15.4 & 14.8 & 16.3 & 12.4 & 2.6 & 6.6 \\
\hline Commercial banks & 14.8 & 14.8 & 14.2 & 16.9 & 13.0 & 2.6 & 7.3 \\
\hline Specialized govt. banks $2 /$ & 16.0 & 24.7 & 25.1 & -- & -- & -- & -- \\
\hline Thrift banks & 18.6 & 13.9 & 13.8 & 11.9 & 7.5 & -0.1 & 0.8 \\
\hline Rural banks & 11.7 & 11.9 & 14.9 & 15.5 & 14.4 & 14.4 & 6.2 \\
\hline \multicolumn{8}{|l|}{ Liquidity ratio $6 /$} \\
\hline Total banking system 1/ & 44.7 & 44.3 & 39.9 & 35.8 & 33.7 & 36.8 & 35.9 \\
\hline Commercial banks & 45.8 & 45.7 & 40.3 & 36.3 & 34.5 & 37.9 & 36.7 \\
\hline Specialized govt. banks 2/ & 44.7 & 34.9 & 39.3 & -- & -- & -- & -- \\
\hline Thrift banks & 36.4 & 38.5 & 38.1 & 32.3 & 26.9 & 27.3 & 29.3 \\
\hline Rural banks & 32.5 & 32.7 & 32.3 & 28.5 & 24.8 & 24.8 & 25.7 \\
\hline
\end{tabular}

Source: Bangko Sentral ng Pilipinas

1/ Banking system data for December 1998 are calculated using June data in the case of the rural banks.

2/ Consolidated with commercial banks after 1995.

3/ After 1994, unadjusted net worth; data reported for June 1998 corresponds to December 1997.

$4 / \mathrm{ROA}=$ Net profit before tax/Total assets.

$5 / \mathrm{ROE}=$ Net profit before tax/capital.

6/Liquid assets/liquid liabilities. 
Table 44. Philippines: Nonperforming Loans of the Banking System, 1993-98

\begin{tabular}{|c|c|c|c|c|c|c|c|c|}
\hline & \multirow[b]{2}{*}{1993} & \multirow[b]{2}{*}{1994} & \multirow[b]{2}{*}{1995} & \multirow[b]{2}{*}{1996} & \multicolumn{2}{|c|}{1997} & \multicolumn{2}{|c|}{1998} \\
\hline & & & & & June & December & June & December \\
\hline & \multicolumn{8}{|c|}{ (In billions of pesos) } \\
\hline Nonperforming loans (NPLs) & 29.9 & 34.0 & 39.0 & 48.4 & 64.5 & 94.9 & 172.0 & 188.8 \\
\hline Commercial banks & 23.8 & 25.1 & 28.0 & 34.2 & 47.9 & 73.7 & 142.7 & 160.0 \\
\hline Thrift banks & 2.7 & 5.5 & 7.0 & 9.5 & 11.3 & 14.9 & 22.5 & 21.4 \\
\hline Rural banks $1 /$ & 3.3 & 3.5 & 4.0 & 4.7 & 5.4 & 6.3 & 6.8 & 7.4 \\
\hline Loan loss provisions & 14.4 & 14.6 & 16.8 & 19.1 & 22.3 & 40.0 & 49.9 & 68.5 \\
\hline Commercial banks & 13.3 & 12.0 & 13.8 & 15.1 & 18.5 & 34.8 & 43.8 & 61.3 \\
\hline Thrift banks & 0.6 & 2.0 & 2.4 & 3.3 & 3.1 & 4.4 & 5.2 & 6.3 \\
\hline \multirow[t]{2}{*}{ Rural banks $1 /$} & 0.5 & 0.6 & 0.7 & 0.7 & 0.7 & 0.8 & 0.9 & 0.9 \\
\hline & \multicolumn{8}{|c|}{ (Percentages) } \\
\hline Ratio of NPLs to total loans & 5.3 & 4.7 & 4.0 & 3.5 & 4.0 & 5.4 & 9.7 & 11.0 \\
\hline Commercial banks & 4.7 & 3.9 & 3.2 & 2.8 & 3.4 & 4.7 & 8.9 & 10.4 \\
\hline Thrift banks & 6.0 & 8.4 & 7.9 & 7.7 & 7.7 & 10.7 & 16.5 & 16.4 \\
\hline Rural banks $1 /$ & 21.3 & 18.2 & 16.1 & 14.1 & 14.6 & 15.4 & 16.7 & 18.0 \\
\hline \multicolumn{9}{|l|}{ Ratio of loan loss provisions } \\
\hline to nonperforming loans & 48.3 & 43.0 & 43.1 & 39.5 & 34.6 & 42.1 & 29.0 & 36.3 \\
\hline Commercial banks & 55.8 & 47.9 & 49.2 & 44.3 & 38.7 & 47.2 & 30.7 & 38.3 \\
\hline Thrift banks & 22.7 & 36.8 & 33.8 & 34.7 & 27.2 & 29.3 & 23.3 & 29.2 \\
\hline Rural banks $1 /$ & 15.4 & 17.7 & 17.2 & 14.4 & 13.3 & 12.7 & 12.7 & 12.7 \\
\hline & \multicolumn{8}{|c|}{ (Percentage growth) } \\
\hline Nonperforming loans (NPLs) & 5.5 & 13.9 & 14.8 & 23.9 & & 96.2 & 166.5 & 98.9 \\
\hline Commercial banks & 6.0 & 5.1 & 11.8 & 22.1 & & 115.6 & 198.2 & 117.0 \\
\hline Thrift banks & -0.6 & 102.5 & 28.2 & 34.3 & & 57.6 & 98.9 & 43.5 \\
\hline Rural banks $1 /$ & 7.1 & 4.9 & 15.0 & 18.2 & & 32.8 & 26.4 & 18.3 \\
\hline Loan loss provisions & 6.7 & 1.4 & 15.1 & 13.4 & & 109.1 & 123.7 & 71.5 \\
\hline Commercial banks & 6.9 & -9.9 & 14.9 & 9.9 & & 129.6 & 136.5 & 76.3 \\
\hline Thrift banks & 3.0 & 228.6 & 17.6 & 38.0 & & 33.4 & 70.7 & 43.0 \\
\hline Rural banks $1 /$ & 6.9 & 20.8 & 11.4 & -0.9 & & 17.2 & 21.1 & 18.6 \\
\hline
\end{tabular}

Sources: Bangko Sentral ng Pilipinas; and Fund staff estimates.

1/ Data for June 1998 correspond to March 1998. 
Table 45. Philippines: Minimum Capital Requirements in the Banking System (In millions of pesos)

\begin{tabular}{|c|c|c|c|c|}
\hline & \multirow{2}{*}{$\begin{array}{c}\text { Before } \\
\text { Dec. } 31,1998 \\
\end{array}$} & \multicolumn{3}{|c|}{ New Requirements } \\
\hline & & Dec. 31,1998 & Dec. 31, 1999 & Dec. 31,2000 \\
\hline Expanded commercial banks & 3,500 & 4,500 & 4,950 & 5,400 \\
\hline Commercial banks & 1,625 & 2,000 & 2,400 & 2,800 \\
\hline \multicolumn{5}{|l|}{ Thrift banks } \\
\hline Within Metro Manila & 200 & 250 & 325 & 400 \\
\hline Outside Metro Manila & 40 & 40 & 52 & 64 \\
\hline \multicolumn{5}{|l|}{ Rural banks } \\
\hline Within Metro Manila 1/ & 20 & 20 & 26 & 32 \\
\hline Cities of Cebu and Davao 1/ & 5 & 5 & 6.5 & 8 \\
\hline Smaller cities and municipalities & $2-3$ & $2-3$ & $2.6-3.9$ & $3.2-4.8$ \\
\hline
\end{tabular}

Source: Bangko Sentral ng Pilipinas

1/ For existing banks only; no new rural banks are presently allowed in Manila, Cebu or Davao. 
Table 46. Selected Asian Countries: Schedule of Provisions for Probable Loan Losses

(Percent of loan amount)

\begin{tabular}{|c|c|c|c|c|c|c|c|c|c|c|}
\hline & \multicolumn{7}{|c|}{ Philippines } & \multirow[b]{3}{*}{ Indonesia } & \multirow[b]{3}{*}{ Korea } & \multirow[b]{3}{*}{ Thailand $1 /$} \\
\hline & \multirow{2}{*}{$\begin{array}{c}\text { Before } \\
12 / 31 / 98\end{array}$} & \multirow{2}{*}{$\begin{array}{c}\text { Before } \\
10 / 1 / 98\end{array}$} & \multicolumn{5}{|c|}{ New requirements } & & & \\
\hline & & & $12 / 31 / 98$ & $4 / 15 / 99$ & $10 / 1 / 98$ & $4 / 1 / 99$ & $10 / 1 / 99$ & & & \\
\hline \multicolumn{11}{|l|}{ Specific loan loss provisions } \\
\hline Especially mentioned loans & 0 & & 2.5 & 5 & & & & 5 & 2 & 2 \\
\hline \multicolumn{11}{|l|}{ Substandard loans } \\
\hline Collateralized & 0 & & 12.5 & 25 & & & & 15 & 20 & 20 \\
\hline Uncollateralized & 25 & & 25 & 25 & & & & 15 & 20 & 20 \\
\hline Doubtful loans & 50 & & 50 & 50 & & & & 50 & 75 & 50 \\
\hline Loss & 100 & & 100 & 100 & & & & 100 & 100 & 100 \\
\hline General Loan Loss Provisions & & 0 & & & 1.0 & 1.5 & 2 & 1 & 0.5 & 1 \\
\hline
\end{tabular}

Source: Bangko Sentral ng Pilipinas

1/ Total provisioning requirements are phased in gradually, starting 12/31/98; provisioning requirements will increase by 20 percent every six months, until full provisioning has been reached by $12 / 31 / 2000$.

2/ Phased in 0.25 percent every six months, starting with 0.25 percent on $12 / 31.98$, reaching I percent on 12/31/2001. 